\title{
1 Individual variation in dispersal, and its sources, shape \\ 2 the fate of pushed vs. pulled range expansions
}

3 Maxime Dahirel ${ }^{1,2, *}$, Chloé Guicharnaud $^{1}$, Elodie Vercken ${ }^{1}$

$4{ }^{1}$ Université Côte d'Azur, INRAE, CNRS, ISA, France

$5{ }^{2}$ Ghent University, Department of Biology, Belgium

6 *corresponding author: maxime.dahirel@yahoo.fr 


\section{Abstract}

9 Ecological and evolutionary dynamics of range expansions are shaped by both dispersal and

10 population growth. Accordingly, density-dependence in either dispersal or growth can

11 determine whether expansions are pulled or pushed, i.e. whether expansion velocities and

12 genetic diversity are mainly driven by recent, low-density edge populations, or by older

13 populations closer to the core. Despite this and despite abundant evidence of dispersal

14 evolution during expansions, the impact of density-dependent dispersal and its evolution on

15 expansion dynamics remains understudied. Here, we used simulation models to examine the

16 influence of individual trait variation in both dispersal capacity and dispersal density-

17 dependence on expansions, and how it impacts the position of expansions on the pulled-pushed

18 continuum. First, we found that knowing about the evolution of density-dependent dispersal at

19 the range edge can greatly improve our ability to predict whether an expansion is (more)

20 pushed or (more) pulled. Second, we found that both dispersal costs and the sources of

21 variation in dispersal (genetic or non-genetic, in dispersal capacity versus in density-

22 dependence) greatly influence how expansion dynamics evolve. Among other scenarios, pushed

23 expansions tended to become more pulled with time only when density-dependence was highly

24 heritable, dispersal costs were low and dispersal capacity could not evolve. When, on the other

25 hand, variation in density-dependence had no genetic basis, but dispersal capacity could evolve,

26 then pushed expansions tended to become more pushed with time, and pulled expansions

27 more pulled. More generally, our results show that trying to predict expansion velocities and

28 dynamics using trait information from non-expanding regions only may be problematic, that

29 both dispersal variation and its sources play a key role in determining whether an expansion is

30 and stays pushed, and that environmental context (here dispersal costs) cannot be neglected.

31 Those simulations suggest new avenues of research to explore, both in terms of theoretical

32 studies and regarding ways to empirically study pushed vs. pulled range expansions.

33 Keywords: density-dependent dispersal; dispersal evolution; individual-based models;

34 expansion velocity; genetic diversity; heritability 


\section{Introduction}

36 By redistributing both genes and individuals in space, often non-randomly, dispersal has the 37 potential to influence many aspects of ecological dynamics (e.g. Jacob et al., 2019; Little et al., 38 2019). Understanding what drives dispersal is especially important in the context of range 39 expansions and climate-driven range shifts, as spread in space is the product of dispersal and 40 population growth dynamics (Lewis et al., 2016). Dispersal is a complex trait with multiple and 41 interacting drivers (Bowler \& Benton, 2005; Matthysen, 2012). Intuitively, one may assume that 42 qualitatively different dispersal or growth functions would therefore lead to qualitatively 43 different expansion dynamics. In the context of range expansions, this has been especially 44 studied with respect to density-dependence. One can indeed differentiate between "pushed" or 45 "pulled" expansions (or maybe more accurately place them on a "pushiness" gradient), based 46 on the density-dependence of dispersal and/or growth (Birzu et al., 2019; Lewis et al., 2016; 47 Stokes, 1976). Pulled expansions are predicted to happen when the product of dispersal and 48 growth is maximal at low densities; spread is then primarily driven by what happens in the low-

49 density edge populations where dispersal or growth are maximal. On the other side of the 50 continuum, pushed expansions should happen when dispersal and/or growth are instead 51 increasing with population density, due to e.g. density-dependent dispersal or Allee effects 52 (Birzu et al., 2019). In that case, the contributions of dispersal and growth at the low-density 53 edge to overall spread are outweighed by what happens in older more populated habitats; 54 expansions are thus "pushed" forward by these older populations. The more pushed an 55 expansion is, the more predictions of spread velocity based only on low-density behaviour will 56 underestimate its true speed (Birzu et al., 2018, 2019; Gandhi et al., 2016, 2019)

57 Evolutionary dynamics during expansions are likely influenced by whether they are pushed or 58 pulled (Miller et al., 2020): pulled expansions are typically predicted to lose genetic diversity 59 faster than pushed ones, as new populations are founded by fewer individuals (Roques et al., 60 2012). On the one side, higher genetic diversity in pushed expansions may mean they are better 61 equipped to successfully adapt to new environmental conditions encountered during expansion 62 (Szúcs et al., 2017). On the other hand, the fact that founding population sizes may be larger at 63 the edge in pushed expansions may mean that spatial sorting processes (Phillips \& Perkins, 2019 
64 and see below) are less selective (Miller et al., 2020). In contrast to this, the traditional view of

65 the pushed/pulled expansion distinction in theoretical works implicitly assumes there is no

66 individual variation, and thus no evolution, in the very traits that generate "pushiness"; that is,

67 the density-dispersal and density-growth functions are the same for all individuals, and remain

68 constant during the expansion.

69 Individual variation in dispersal and dispersal-related traits is very common and now well 70 documented (Bowler \& Benton, 2005; Cote et al., 2017; Jacob et al., 2019; Ronce \& Clobert,

71 2012), and while a lot of questions remain, it is clear that a non-negligible fraction of this

72 variation is genetic (Saastamoinen et al., 2018). Following Cote et al. (2017)'s terminology, we

73 can distinguish individual variation in dispersal capacity/ability, which is linked to variation in

74 enabling traits, making dispersal possible at all (e.g. presence/ absence of wings, Simmons \&

75 Thomas, 2004) or enhancing traits (e.g. body condition or wing/leg length, Baines et al., 2019;

76 Baines \& McCauley, 2018), from individual variation in context-dependence, which can be linked

77 to matching traits that do not alter dispersal capacity per se, but lead to non-random dispersal

78 in response to experienced environmental conditions.

79 We also know that this individual variation can influence spread dynamics (Miller et al., 2020).

80 Genetic differences in dispersal traits can fuel evolution at the expanding range edge by spatial 81 sorting, as at each generation, individuals with spread-facilitating traits are more likely to 82 advance to new habitats and reproduce there (Phillips \& Perkins, 2019; Shine et al., 2011). The 83 effects of spatial sorting on spread velocity are well documented, thanks to both iconic natural 84 examples (e.g. Phillips et al., 2006) and experiments reshuffling individuals to cancel out the 85 effects of spatial evolution (Ochocki \& Miller, 2017; Weiss-Lehman et al., 2017). Because 86 dispersal is often associated with other traits in syndromes (Ronce \& Clobert, 2012), changes in 87 many traits along expansions are potentially linked with spatial sorting (Chuang \& Peterson, 88 2016).

89 However, these studies have so far mostly focused on variation in dispersal capacity (or the 90 underlying enabling or enhancing traits). As a result, and despite some indications that 91 individual variation in density-dependence itself may be subject to selection during range 
92 expansions (Dahirel, Bertin, Calcagno, et al., 2021; Fronhofer et al., 2017; Mishra et al., 2020;

93 Weiss-Lehman et al., 2017), we mostly don't know how the structure of individual variation in

94 dispersal influences whether an expansion is and stays pushed or pulled. Indeed, the one

95 theoretical study on the subject, which showed that genetic variation leads pushed expansions

96 to become pulled with time, was focused on Allee effects only (Erm \& Phillips, 2020). In the

97 context of dispersal, theory developed outside of the pushed/pulled framework suggests that

98 dispersal can become more density-independent at range edges (Travis et al., 2009), which

99 would agree with Erm and Phillips (2020)'s predictions. However, that theoretical study and

100 others typically allow only positive density-dependent dispersal to evolve, when negative

101 density-dependent dispersal is just as likely in nature (Harman et al., 2020). This may explain

102 mismatches with some of the few existing experimental studies (Dahirel, Bertin, Calcagno, et al.,

103 2021; Fronhofer et al., 2017; Mishra et al., 2020). More importantly, the fact that dispersal

104 becomes on average more density-independent across the range of densities tells us nothing in

105 itself about the difference between average/maximal dispersal and specifically low-density

106 dispersal. It is that difference which should a priori matter for the position of an expansion on

107 the pushed/pulled gradient.

108 In this context, we used individual-based simulations to examine how genetic and non-genetic

109 sources of inter-individual trait variation influence the evolution and maintenance of pushed vs

110 pulled expansion dynamics. We focused on dispersal variation, rather than on Allee effects as

111 many studies on pushed expansions did so far (e.g. Birzu et al., 2018; Erm \& Phillips, 2020;

112 Gandhi et al., 2016; Roques et al., 2012; but see Birzu et al., 2019) for two reasons. First, while

113 there are reasons to believe the broad qualitative results hold whether pushed expansions are

114 caused by Allee effects or dispersal (Birzu et al., 2019), this still needs to be confirmed,

115 especially as evolution of dispersal-density reaction norms would interact with selection for

116 higher dispersal at range edges. Second, although there are no directly comparable systematic

117 syntheses, available evidence suggests positive density-dependent dispersal is more frequent

118 than (demographic) Allee effects in nature (Gregory et al., 2010; Harman et al., 2020),

119 something that is not (yet) reflected in the literature on pushed expansions. We examined how

120 dispersal influences whether expansions are pushed or pulled by drawing on several lines of 
121 evidence, including genetic diversity information (Gandhi et al., 2016, 2019). We determined

122 how the sources of dispersal variation (genetic vs. non genetic, in dispersal capacity or dispersal

123 matching traits), influence the evolution of "pushiness". Finally, we analyzed how dispersal

124 mortality interact with these sources of variation to shape dispersal evolution during range

125 expansions. In non-expanding populations at least, higher costs are predicted to favour more

126 positive density-dependent dispersal (Rodrigues \& Johnstone, 2014; Travis et al., 1999), which

127 would influence the evolution of pushed vs. pulled dynamics.

\section{Methods}

129 Our discrete-time and discrete-space simulation model was written in NetLogo (Wilensky, 1999)

130 version 6.2.0. We interacted with the model and designed our experiments using the nlrx $R$ 131 package (Salecker et al., 2019). We provide below a summarised description of the model, 132 before presenting the simulation experiments we ran using it. A detailed description of the

133 simulation model using the ODD protocol (Grimm et al., 2010; Grimm et al., 2020) can found in

134 Supplementary Material S1, and a copy is attached with the model and analysis code (Data 135 availability).

\section{Summary model description}

137 The overall purpose of our model was to understand how dispersal trait distribution and 138 dispersal evolution shape evolutionary dynamics during range expansions, with a focus on 139 density-dependence and the emergence of pushed vs pulled dynamics. Briefly, the model 140 describes the dynamics of asexual haploid individuals spreading in a linear discrete landscape, 141 with their growth dynamics shaped by a Ricker model and their dispersal by Kun and Scheuring 142 (2006)'s model (Fig. 1). Dispersal and growth are both stochastic. Individuals can vary or not in

143 traits shaping dispersal, and that variation can be more or less heritable. Dispersal is limited to 144 the nearest neighbours, and dispersal costs can be imposed in the form of a mortality risk. 
A

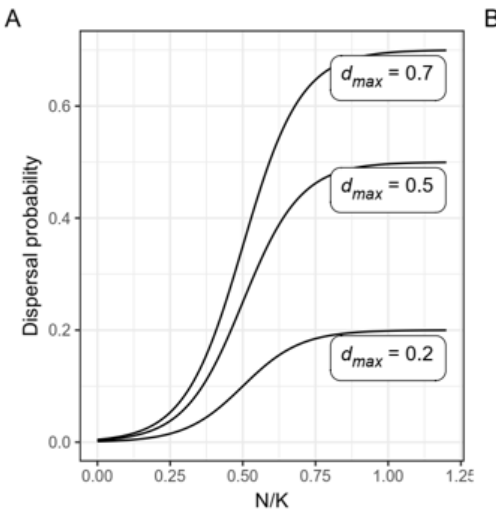

D

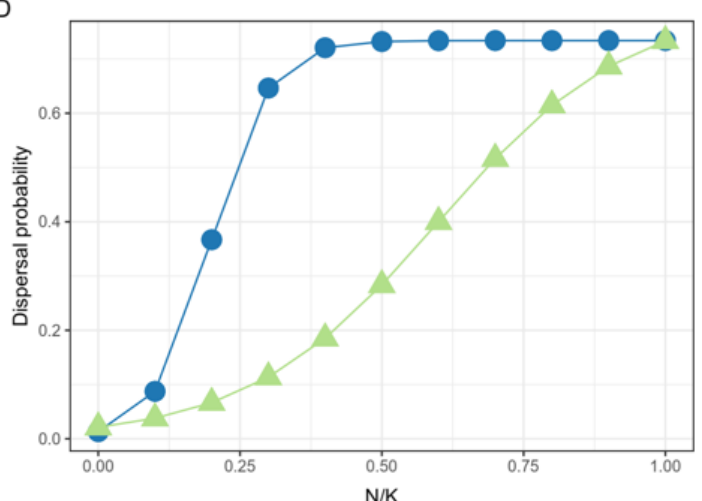

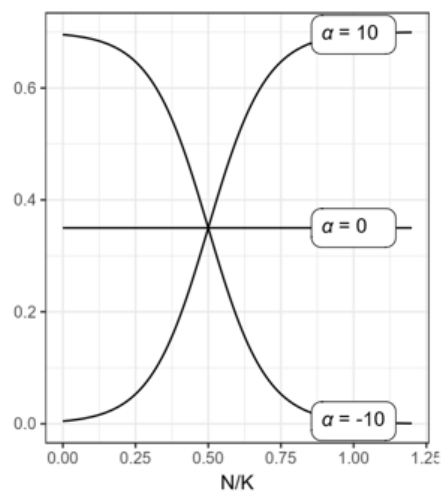

E

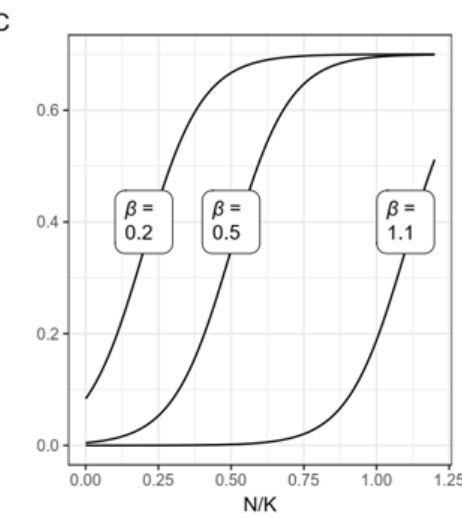

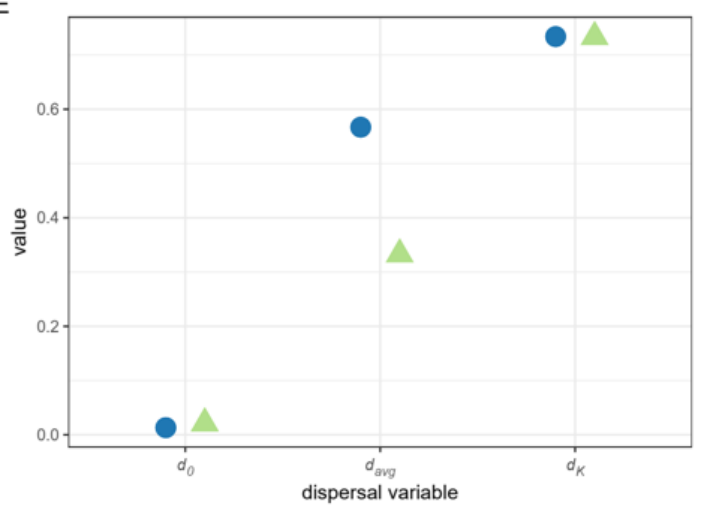

146 Figure 1. Illustration of the dispersal function used in the individual-based model (based on Kun 147 \& Scheuring, 2006) and some of its properties. A, B, C: effect of changing one of the three 148 underlying variables while keeping the other two constant. Values when kept constant: $d_{\max }=$ $1490.5, \alpha=10, \beta=0.5$. D: illustration of the sampling points used to estimate $d_{\text {avg }}$ for two 150 example functions, E: effect of the function general shape on $d_{\text {avg }}$. Note how two functions can 151 have (nearly) the same $d_{0}$ and $d_{K}$ (and therefore the same density-dependence $\Delta_{K-0}$ ), while 152 differing in $d_{\text {avg }}$ (and therefore in $\Delta_{\text {avg-0 }}$ ).

153

154 The model world in which our virtual species expands is one-dimensional, has closed 155 boundaries, and is made of discrete patches in which individuals live and between which they 156 may disperse. Patches, which form the spatial units of the model, are characterized by their 157 location $x$ (integer $\geq 0$ ), by their current population size $N$, and by their carrying capacity $K$, the 158 latter being equal and constant among all patches.

159 Individuals each possess three dispersal traits, the maximal dispersal rate $d_{\max }$, the slope $\alpha$, and 160 the midpoint $\beta$. Individual dispersal probability then depends on these traits and local 161 population density $N$ at the start of the dispersal phase (Fig. 1, Kun \& Scheuring, 2006). 
162 Following the terminology in Cote et al (2017), variation in $d_{\max }$ can be interpreted as encoding 163 variation in dispersal enhancing or enabling traits, while variation in $\alpha$ or $\beta$ corresponds to 164 variation in dispersal matching traits. We allow $\beta$ to be negative instead of being constrained to 165 be always $\geq 0$, as this is the most convenient way to generate flat dispersal-density functions 166 (see detailed discussion in Supplementary Materials S1 and S2). To allow for genetic and nongenetic variation, each trait is actually the (transformed) sum of an underlying additive genetic

168 component $\left(\operatorname{logit}\left(d_{\max }\right)_{[a]}, \alpha_{[a]}, \beta_{[a]}\right)$ and an underlying noise/residual part $\left(\operatorname{logit}\left(d_{\max }\right)_{[r]}\right.$, $\left.169 \alpha_{[r]}, \beta_{[r]}\right)$. To track neutral genetic diversity, individuals also possess a neutral locus $\gamma$ at which 170 two allelic values are possible (0 or 1$)$.

171 Once we know the $d_{\max }, \alpha$ and $\beta$ of an individual, we additionally calculate five values at the 172 individual level (Fig. 1D-E):

$173-d_{0}$, the hypothetical dispersal rate at $N=0$. The theoretical distinction between pulled and pushed expansions hinges on whether expansions move as fast as expected from $d_{0}$ or faster (e.g. Birzu et al., 2019);

- $d_{K}$, the expected dispersal rate at $N=K$;

- $d_{a v g}$, the average dispersal over the range of densities $0-K$. For computational reasons, we approximate it by calculating $d_{N}$ every $0.1 K$ from 0 to $K$;

- two measures of the strength of density-dependence $\Delta_{K-0}=d_{K}-d_{0}$ and $\Delta_{a v g-0}=$ $d_{\text {avg }}-d_{0}$. The former is independent of the shape of the dispersal function between 0 and $K$, while the latter accounts for it at least in part.

182 The model is initialised by releasing $K$ adult individuals that have not yet dispersed nor 183 reproduced in the patch $x=0$. Initial values for the genetic and non-genetic components of 184 individual traits are drawn from Normal distributions, with the value of initial heritability $h^{2}$ 185 used to determine how initial phenotypic variance is partitioned between genetic and non186 genetic components (see Virtual experiment design below and Table 1 for the means and 187 variances). Initial allelic values for the neutral locus are drawn from Bernoulli(0.5). The world is 188 set to be long enough that the expansion never runs out of patches. As we assume stepping- 
189 stone dispersal (dispersers only move one patch) and the expansion advances in one direction,

190 this means, in practice, that the world can be any number of patches as long as it is larger than

191 the number of generations in a run (for run duration in our experiment, see Virtual experiment

192 design).

193 Generations are non-overlapping, and every time step, the life cycle unfolds as follows:

194 - Individuals are counted, providing information about patch population sizes $N$ for 195 dispersal;

- Dispersal: Newly adult individuals may disperse with a probability $d$ depending on their individual traits $\left(d_{\max }, \alpha, \beta\right)$ and local population size $N: d=\frac{d_{\max }}{1+\exp \left(-\alpha\left(\frac{N}{K}-\beta\right)\right)}$ (Fig. 1, Kun \& Scheuring, 2006). Individuals that do disperse die with a probability $m$; if they survive, they settle in one of the immediate neighbouring patches, randomly chosen.

- Individuals are re-counted post-dispersal, updating population sizes $N$ for the reproduction phase;

- $\quad$ Reproduction: Each remaining adult then produces $F$ juveniles, with $F \sim \operatorname{Poisson}(\lambda)$ and the mean fecundity $\lambda$ based on a Ricker model: $\lambda=\mathrm{e}^{r_{0}(1-N / K)}$, where $r_{0}$ is the lowdensity growth rate. Juveniles are born in the patch currently occupied by their parent, and directly inherit their genetic values for dispersal-related and neutral loci. Their values for the noise part of the dispersal traits are redrawn at random.

- $\quad$ Death and end of cycle: All adults die; juveniles then become adults.

208 To facilitate further analyses, a series of patch-level summaries of traits are computed every 209 generation. In this study, we are particularly interested in the means of $d_{\text {max }}, d_{0}$, the density210 dependence metrics $\Delta_{K-0}, \Delta_{a v g-0}$, and the neutral allele frequencies (all collected right before 211 the dispersal phase). 


\section{Simulation experiments and analyses}

\section{Virtual experiment design}

214 We tested all possible combinations of the starting parameters described in Table 1, with the 215 exception of the effectively redundant ones (namely heritabilities $h^{2}>0$ when all phenotypic 216 variances were 0), resulting in 270 scenarios. We ran expansions for 120 generations, and 217 "observed" them every 20 generations to save memory. We replicated each scenario 10 times. 218 Besides the extreme $h^{2}=0$ and $h^{2}=1$ cases, the "intermediate" level of initial heritability was 219 set to 0.33 to roughly match "average" heritabilities seen in dispersal traits (Saastamoinen et al., 220 2018). The growth rate $r_{0}$ was constant and set to a value that is within the range of plausible 221 values for insects (Hassell et al., 1976), and worked well in previous simulations (Dahirel, Bertin, 222 Haond, et al., 2021). $K$ was chosen to match previous simulations (Dahirel, Bertin, Haond, et al., 223 2021; Haond et al., 2018), and is within an order of magnitude of typical population sizes seen 224 during experimental range expansions in plants or invertebrates (e.g. Dahirel, Bertin, Haond, et 225 al., 2021; Ochocki \& Miller, 2017; Van Petegem et al., 2018; Williams \& Levine, 2018). The value 226 for the initial phenotypic variance for $\operatorname{logit}\left(d_{\text {max }}\right)$ was chosen because a $\operatorname{Normal}(\mu=0, \sigma=$ 227 1.5) distribution on the logit scale leads to an approximately uniform distribution over most of 228 the [0;1] range on the proportion scale. The non-zero starting values for the initial mean slope $\alpha$ 229 were chosen based on a key property of the logistic function: $d$ goes from $0.05 \times d_{\max }$ to $2300.95 \times d_{\max }$ over an interval of width $\simeq 6 /|\alpha|$ centered on $\beta$ (see e.g. Börger \& Fryxell, 2012). 231 Setting initial $|\alpha|$ to be 6 means that interval is of width $K$. The initial phenotypic variance for $\alpha$ 232 was chosen so that the mean initial difference between replicates with $\alpha=0$ and replicates 233 with $\alpha \neq 0$ was 1 standard deviation; a similar argument was used to set the initial phenotypic 234 variance for the midpoint $\beta$. 
Table 1. Summary of global parameters used in our simulation model, and their possible values.

\begin{tabular}{cll}
\hline Parameter name & Description & Initial values \\
\hline$m$ & dispersal mortality & $0.1 ; 0.5 ; 0.9$ \\
$h^{2}$ & initial trait heritability & $0 ; 0.33 ; 1$ \\
$r_{0}$ & mean low-density growth rate & $\log (5)$ \\
$K$ & patch carrying capacity & 250 \\
$\bar{d}_{\max }$ & initial average $d_{\text {max }}$ & 0.5 \\
$\bar{\alpha}_{t=0}$ & initial average relative slope parameter of the dispersal-density function & $-6 ; 0 ; 6$ \\
$\bar{\beta}_{t=0}$ & initial average midpoint of the dispersal-density function & $0 ; 0.5 ; 1$ \\
$V_{P\left[\operatorname{logit}\left(d_{\max }\right)\right]}$ & initial phenotypic variance for $d_{\text {max }}$ (on the logit scale) & $0 ; 1.5^{2}$ \\
$\left(V_{P[\alpha]}, V_{P[\beta]}\right)$ & vector of initial phenotypic variances for $\alpha$ and $\beta$ & $(0,0) ;\left(6^{2}, 0.5^{2}\right)$
\end{tabular}

238 We repeated the entire experimental design and analyses with a lower growth rate $\left(r_{0}=\right.$ $239 \log (1.5))$ to determine whether our results on dispersal evolution were sensitive to overall 240 fecundity. Most of our key results (i.e. results pertaining to density-dependence) are upheld; 241 deviations to the results observed when $r_{0}=\log (5)$ are briefly described in Results and 242 Discussion.

243 Analyses

244 All analyses were done using R, version 4.1.2 (R Core Team, 2021). Simulated data handling, 245 analyses and plotting relied mostly on the mgcv (Wood, 2017), gratia (Simpson, 2021), ordinal 246 (Christensen, 2019), MuMIn (Bartoń, 2020) and patchwork (Pedersen, 2020) packages, as well 247 as the tidyverse suite of packages (Wickham et al., 2019).

248 We are interested here in how traits evolve at the range front, i.e. the area at the edge of the 249 expansion where a density gradient is present (e.g. Lewis et al., 2016), and how this influences 250 expansion dynamics. This means we first need to define what patches belong to the range front. 251 Based on plots showing the distribution of population sizes as a function of distance to the 252 farthest-forward population (Supplementary Material S3), we decided all patches less than 5 
253 patches from the limit of the expansion belong to the range front for our purposes. Patches

254 closer to the core typically have already reached an equilibrium density (which may or may not

255 be $K$, depending on the interplay between growth and dispersal in the core; Supplementary

256 Material S3). For each replicate expansion, we used weighted averages (based on population

257 size at the time of trait measurement) to average trait values across front patches before 258 analyses. We ran separate sets of statistical models on runs with $r_{0}=\log (5)$ and runs with $r_{0}=$ $259 \log (1.5)$.

\section{Expansion velocity}

261 Broadly speaking, and unless $r_{0}$ is negative (strong Allee effect, Courchamp et al., 2008), theory 262 predicts that the long run velocity $v$ of an expansion can be described as $v=p \times v_{F}=$ $263 p \times f\left(d_{0}, r_{0}\right)$, where $v_{F}$ is the velocity expected for a pulled expansion with these $r_{0}$ and $d_{0}$ 264 (Birzu et al., 2018, 2019; Gandhi et al., 2016; Lewis et al., 2016; Wang et al., 2019). What we 265 term $p$ here is a measure of "pushiness", and increases the more growth or dispersal are positively density dependent (Birzu et al., 2018, 2019). Although analytical formulas for $v_{F}$ exist

267 in many situations (Wang et al., 2019), they are mostly designed for cases when population 268 densities are high and continuous, rather than counts of discrete individuals. While the resulting 269 bias may be small to negligible in some scenarios (Dahirel, Bertin, Haond, et al., 2021; Haond et 270 al., 2018), the approximations can become important in some cases, for instance when 271 stochasticity is high (e.g. Hallatschek \& Korolev, 2009), and available corrections are not always 272 practical to implement.

273 To circumvent these issues and clearly separate the effect of $d_{0}$ from the effect of density274 dependence, we decided instead to analyse expansion velocities using Generalized Additive 275 Models (GAMs). This allowed us to describe expansion velocities using very flexible non-linear 276 functions of dispersal traits (growth rates being identical across all replicates), without having to 277 choose ourselves the "right" formula. We compared 7 GAMs using $\mathrm{R}^{2}$ as model performance 278 criterion (comparisons based on AICc led to the same interpretations, with the best model 279 based on $\mathrm{R}^{2}$ obtaining an AICC weight $>0.99$ ). In all models we use the number of new patches 280 populated between generations 100 and 120 as our "expansion velocity" response variable, as it 
281 was both after evolutionary changes were mostly complete and after velocity had seemingly reached an equilibrium (Supplementary Material S4). Since our landscape is made of discrete 283 patches and the maximal possible expansion speed is one patch/generation, we analysed these data using binomial GAMs. There were some indications of underdispersion, likely because the probability of the expansion moving forward was not independent from one generation to the next, due to temporal autocorrelation in population sizes. More complex models accounting for this underdispersion gave similar conclusions (see archived code in Data availability for an example); we here only present the binomial GAMs results for simplicity. The simplest model, used as a null model of sorts, assumed expansion speed depended only on dispersal mortality (a categorical variable with three levels, Table 1) and not on any dispersal traits. The other models were divided equally into models that used trait information collected before the range expansion started, versus models that used trait information collected at generation 100, 293 i.e. after genetic variation at the front was mostly spent (Supplementary Material S4). In both 294 cases we analysed:

- (a) a model assuming velocity depended on mortality, a spline effect of mean $d_{0}$ at the range edge, and their interaction;

- (b) a model accounting for density-dependence by adding to (a) a spline effect of the mean difference $\Delta_{K-0}$ between $d_{0}$ and $d_{K}$ at the range front (and its interaction with mortality);

- (c) a model accounting for density-dependence by adding to (a) a spline effect of the mean difference $\Delta_{a v g-0}$ between $d_{0}$ and $d_{a v g}$ at the range front (and again its interaction with mortality).

\section{Genetic diversity}

304 Another key aspect of pushed vs. pulled dynamics is the fate of genetic diversity, which is lost

305 faster in pulled expansions compared to the "equivalent" pushed expansions (same $r_{0}$ and $d_{0}$ ) 306 (Birzu et al., 2019). To better understand this, we analysed the effect of dispersal traits on time 307 to genetic fixation, i.e. the first generation at which only one of the two neutral alleles is 
308 detected in the range front. Since we have grouped "survival" times, as we only collected 309 information every 20 generations to save memory, we used cumulative ordinal regression

310 models to analyse these data. In these models, the response variable is a categorical variable 311 where the categories are ordered (here, along the time axis), and the category an observation

312 belongs to can be seen as a coarse reflection of an unmeasured latent continuous response 313 (here the actual time to fixation; Bürkner \& Vuorre, 2019). When these models are fit with a 314 complementary log-log link, they correspond to a proportional hazards model (e.g. Bürkner \& 315 Vuorre, 2019; Tutz \& Schmid, 2016). We tested the same seven model structures as for velocity, 316 although covariates were this time included "as is" and not as non-linear splines. Models were 317 again compared using $\mathrm{R}^{2}$ as criteria. More accurately, we used a pseudo- $\mathrm{R}^{2}$ based on a linear 318 regression between observed and mean predicted category (comparisons based on AICc led 319 again to the same interpretations, with the best model based on $\mathrm{R}^{2}$ again obtaining an AICC 320 weight $>0.99)$. When analysing simulations with $r_{0}=\log (5)$, two simulations out of 2700 were 321 excluded due to not having advanced (meaning they did not have any front to analyse). When 322 analysing simulations with $r_{0}=\log (1.5), 64$ replicate runs out of 2700 (so $2.4 \%$ ) were 323 excluded, 21 because they did not advance, 43 because all populations went fully extinct before 324 the end of the run.

\section{Trait evolution}

326 Finally, to understand how trait evolution was influenced by initial conditions and dispersal 327 costs, we focused on two traits: the density-dependence $\Delta_{a v g-0}$ and the dispersal capacity $328 d_{\max }$. For $\Delta_{a v g-0}$, we displayed the change in mean value in front patches after 100 generations 329 as a fonction of the starting mean value for each combination of mortality, initial heritability, 330 presence/absence of individual variation in dispersal traits at the start of the expansion, and 331 used linear regressions to highlight the overall trends. For $d_{\text {max }}$, we simply displayed the mean 332 value at the front, since the initial mean value was the same (0.5) across all replicates. 


\section{Results}

334 Results for $r_{0}=\log (5)$

335 For both velocity and neutral genetic diversity, the best statistical models were the ones 336 including information about both $d_{0}$ and density-dependence (Fig. 2). For velocities, $R^{2}$ was 337 highest when we used traits after evolution to predict velocities, while for neutral diversity it 338 was highest when we used traits measured before expansion (Fig. 2).

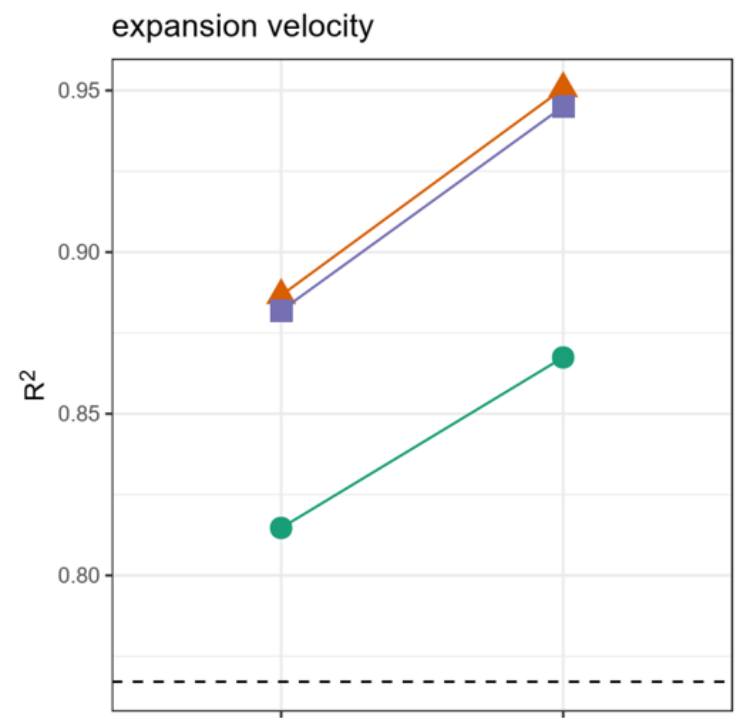

before evolution $(t=0) \quad$ after evolution $(t=100)$ when are trait covariables sampled?

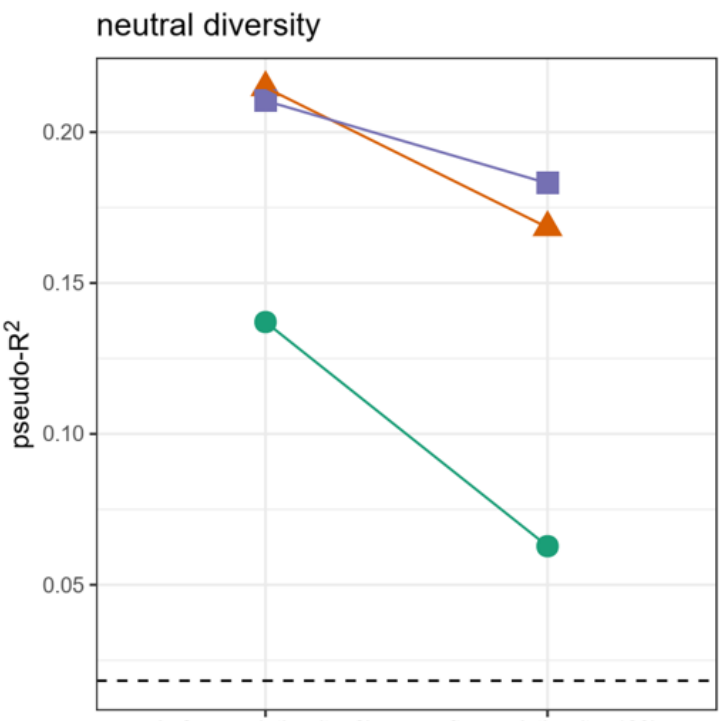

before evolution $(\mathrm{t}=0) \quad$ after evolution $(\mathrm{t}=100)$ when are trait covariables sampled?
Density dependence?

- ignored

$\triangle \Delta_{\text {avg-0 }}$ (shape-dep.)

- $\Delta_{K-0}$ (shape-indep.)

340 Figure 2. $R^{2}$ of the different models used to analyse simulations outputs with respect to 341 expansion velocity in the last 20 generations (left) and time to loss of neutral genetic diversity in 342 the range front (right) (case where $r_{0}=\log (5)$, see Supplementary Figures S5.1 and S5.2 for 343 the case where $r_{0}=\log (1.5)$ ). Dashed line: $\mathrm{R}^{2}$ for the "baseline" model containing only dispersal 344 mortality (all models include dispersal mortality, and its interactions with the other covariates 345 when present).

347 Based on the best model, expansion velocity increased with $d_{0}$ (Fig. 3), and for a given $d_{0}$, 348 expansions with positive density-dependent dispersal were consistently faster (Fig. 3). Increased 349 mortality costs decreased velocity overall and reduced the effect of $d_{0}$ and density-dependence 350 on velocity, but did not change the overall direction of the effect. 
bioRxiv preprint doi: https://doi org/10.1101/2022.01.12 476009. this version posted January 13,2022 . The copyright holder for this preprint (which was not certified by peer review) is the author/funder, who has granted bioRxiv a license to display the preprint in perpetuity. It is made available under aCC-BY 4.0 International license.
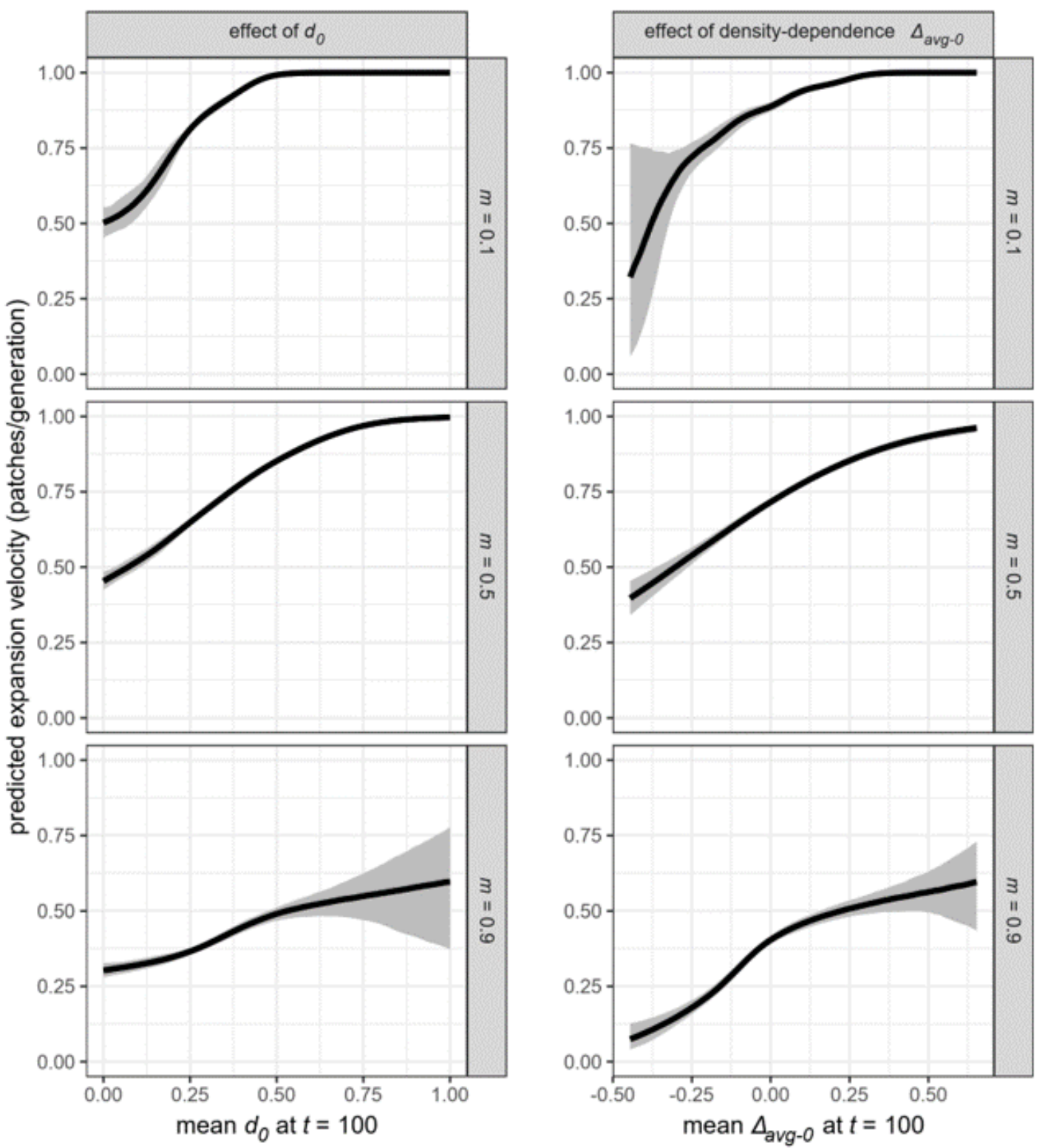

Figure 3. Predicted effect of $d_{0}$ (left) and density-dependence $\Delta_{\text {avg-0 }}$ (right) on expansion velocity, depending of dispersal mortality (case where $r_{0}=\log (5)$, see Supplementary Figure S5.3 for the case where $\left.r_{0}=\log (1.5)\right)$. Predictions are based on the best model Fig. 2; predictions for the effect of $d_{0}$ are made assuming no density-dependence $\left(\Delta_{\text {avg-0 }}=0\right)$, and predictions for the effect of $\Delta_{a v g-0}$ are made setting $d_{0}$ to its average value in the simulated dataset.

359 Higher $d_{0}$ at the start of an expansion led to faster loss of neutral genetic diversity at the range 
bioRxiv preprint doi: https://doi.org/10.1101/2022.01.12.476009; this version posted January 13,2022 . The copyright holder for this preprint (which was not certified by peer review) is the author/funder, who has granted bioRxiv a license to display the preprint in perpetuity. It is made available under aCC-BY 4.0 International license.

361 showed positive density-dependent dispersal maintained neutral diversity at the edge longer 362 than those that did not, but only when dispersal costs were low or intermediate (Fig. 4). This 363 effect of density-dependence was however reversed when dispersal costs were high (Fig. 4).
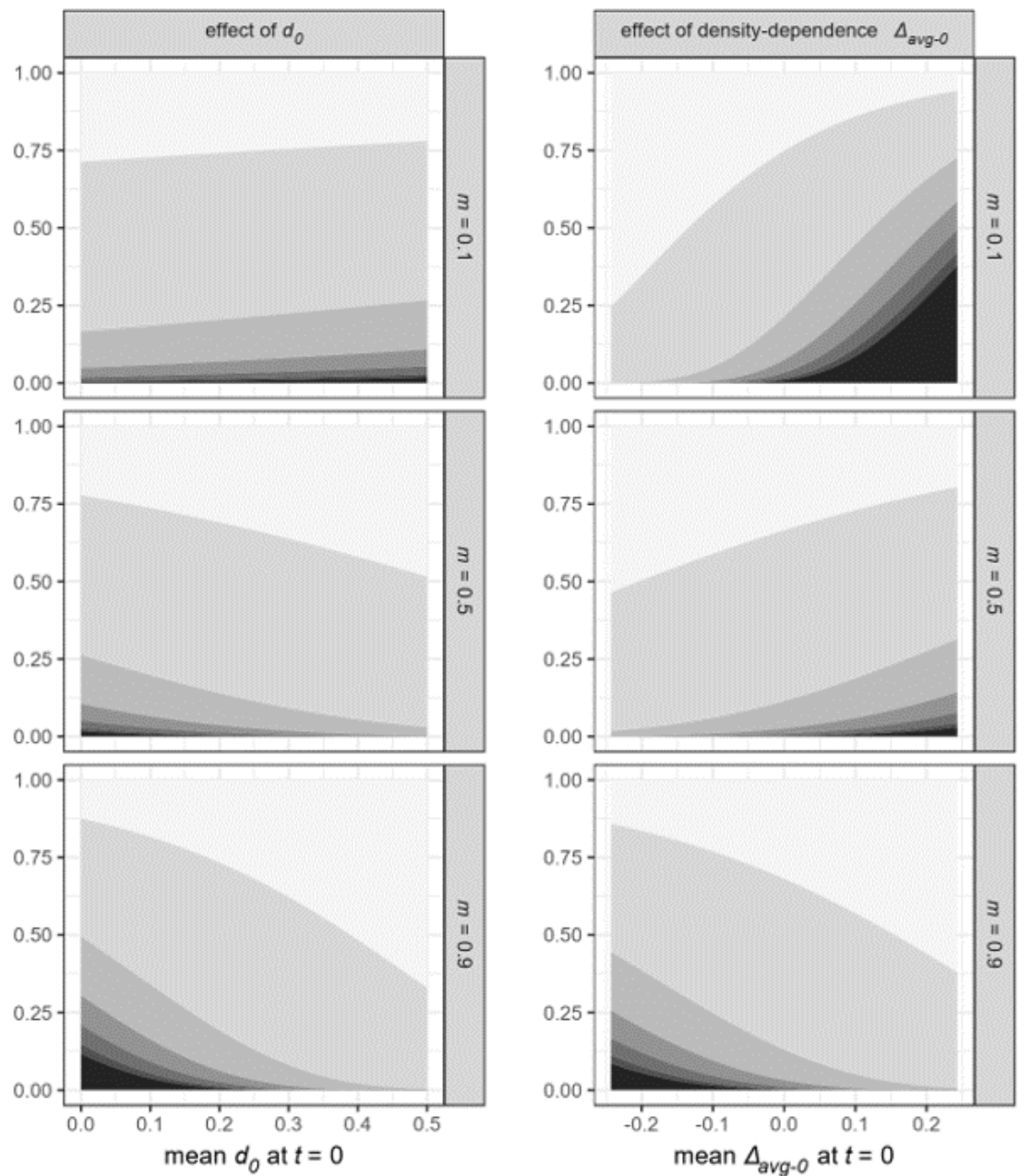

generations before allele fixation:

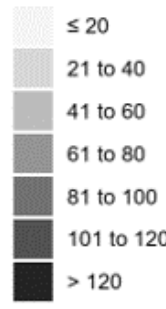

365 Figure 4. Predicted effect of $d_{0}$ (left) and density-dependence $\Delta_{\text {avg-0 }}$ (right) on the loss of neutral diversity at the range front, depending of dispersal mortality (case where $r_{0}=\log (5)$, see Supplementary Figure S5.4 for the case where $\left.r_{0}=\log (1.5)\right)$. Darker shades of grey indicate later fixation times. Predictions are based on the best model Fig. 2; predictions for the effect of $d_{0}$ are made assuming no density-dependence $\left(\Delta_{\text {avg-0 }}=0\right)$, and predictions for the effect of $\Delta_{a v g-0}$ are made setting $d_{0}$ to its average value in the simulated dataset.

372 When $d_{\max }$ was allowed to evolve, it consistently evolved towards higher values at the range edge, whether or not other traits did evolve (Fig. 5). The strength of this response depended on 
374 heritability (evolutionary changes in $d_{\max }$ were larger the more the trait was heritable) and 375 mortality (changes were smaller the more dispersal was costly). Variance in outcomes was 376 important and, although limited, cases of evolutionary decreases in $d_{\text {max }}$ could be observed 377 (Fig. 5); they were more frequent with increased dispersal costs.
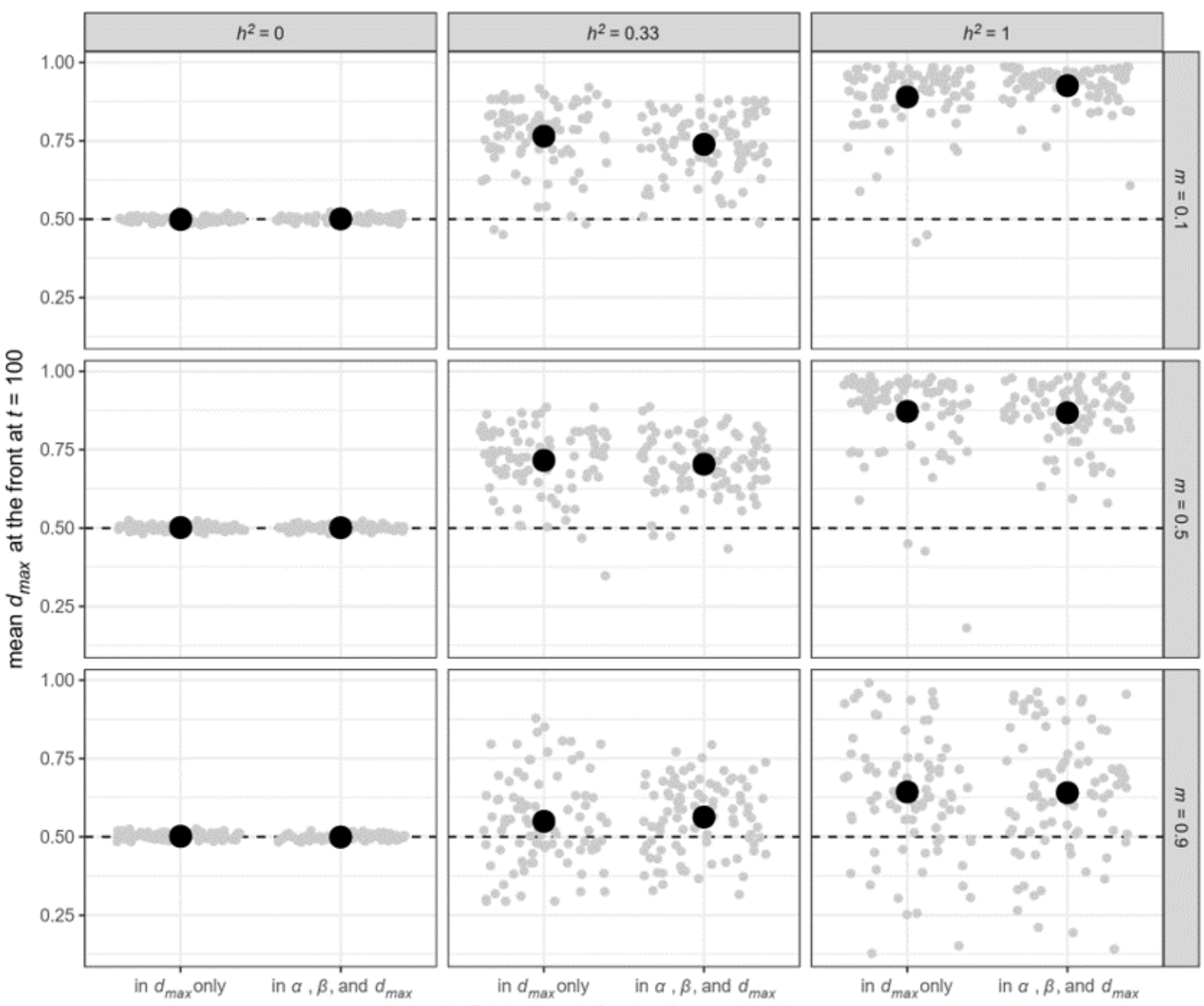
individual variation in dispersal traits

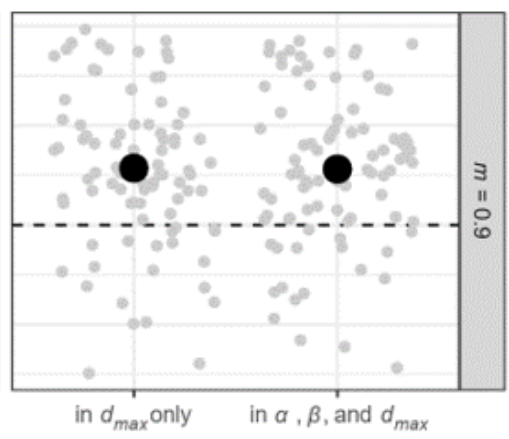

379 Figure 5. Evolutionary changes in dispersal capacity $d_{\max }$ at the range front as a function of 380 sources of phenotypic variation and dispersal costs (case where $r_{0}=\log (5)$, see 381 Supplementary Figure S5.5 for the case where $r_{0}=\log (1.5)$ ). Grey dots are individual 382 replicates, with the average values overlaid in black. Replicates with no individual variation in $383 d_{\max }$ at $t=0$ are not displayed.

385 When only context-dependency $(\alpha$ and $\beta$ ) were allowed to evolve, changes in the density386 dependence $\Delta_{a v g-0}$ depended on heritability and dispersal costs, but overall leaned towards 
387 the evolution of more positive density-dependence, unless heritability was high and mortality 388 costs were low (Fig. 6A). In that specific case, initially pushed expansions (positive density389 dependence $\Delta_{\text {avg-0 }}$ ) tended to lose that density-dependence with time (Fig. 6A, top right). 390 When only $d_{\max }$ was allowed to evolve, density-dependence tended to become stronger after 391 evolution, with initially negative $\Delta_{a v g-0}$ becoming more negative, and initially positive $\Delta_{\text {avg-0 }}$ 392 becoming more positive (Fig. 6 B). When both $d_{\max }$ and context dependence were allowed to 393 evolve, the general trend was to the evolution of positive density-dependence (Fig. 6C).

\section{A: Individual variation in $\alpha$ and $\beta$}
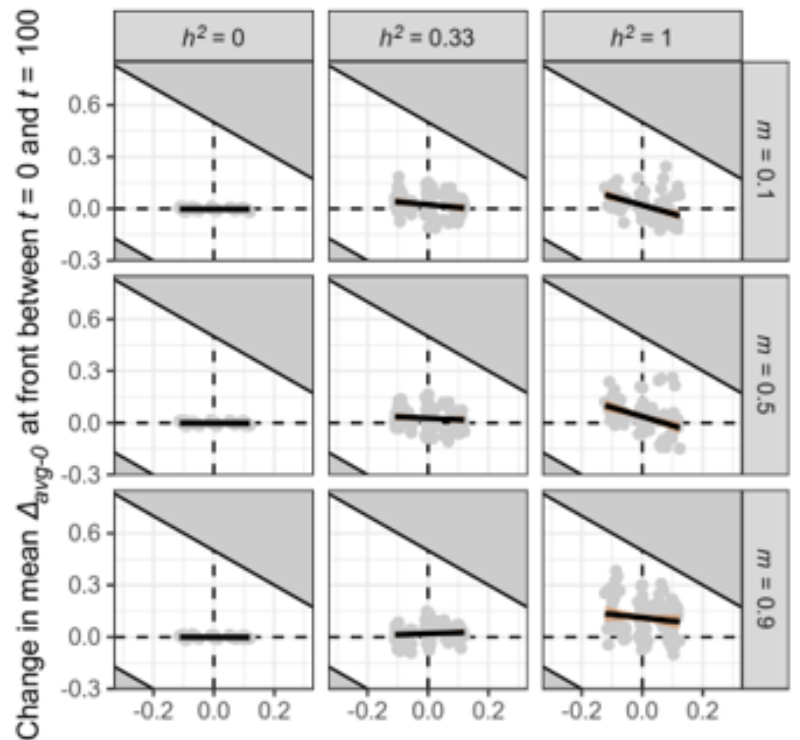

B: Individual variation in $d_{\max }$

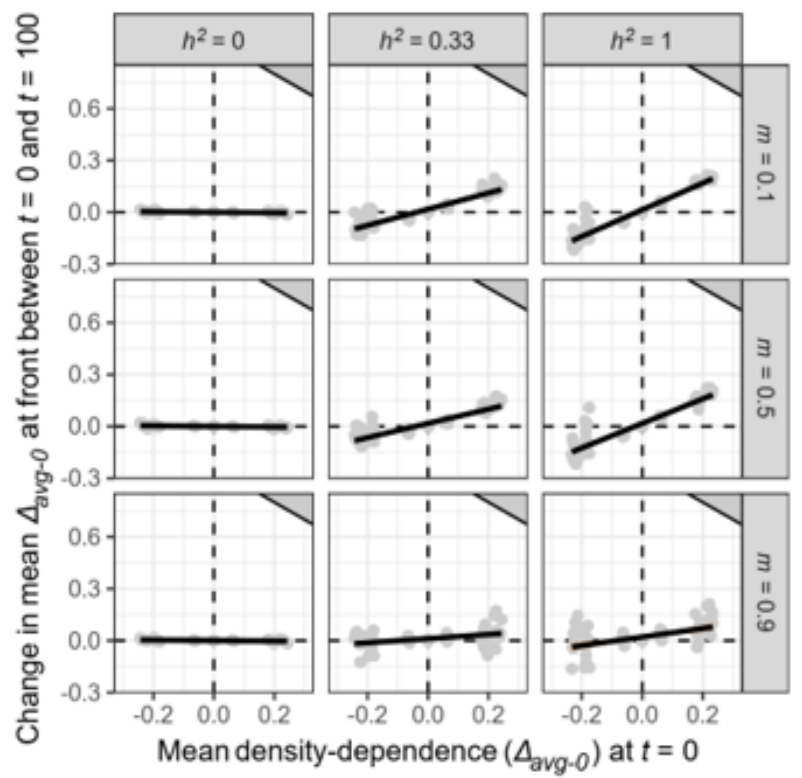

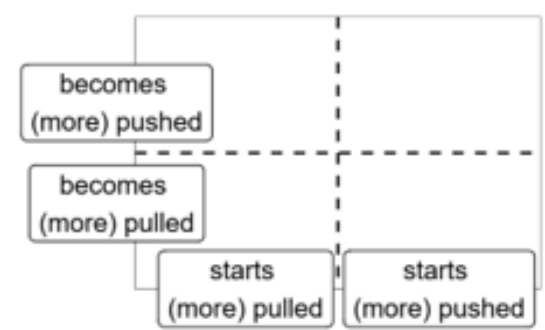

$\mathrm{C}$ : Individual variation in $\alpha, \beta$, and $d_{\max }$

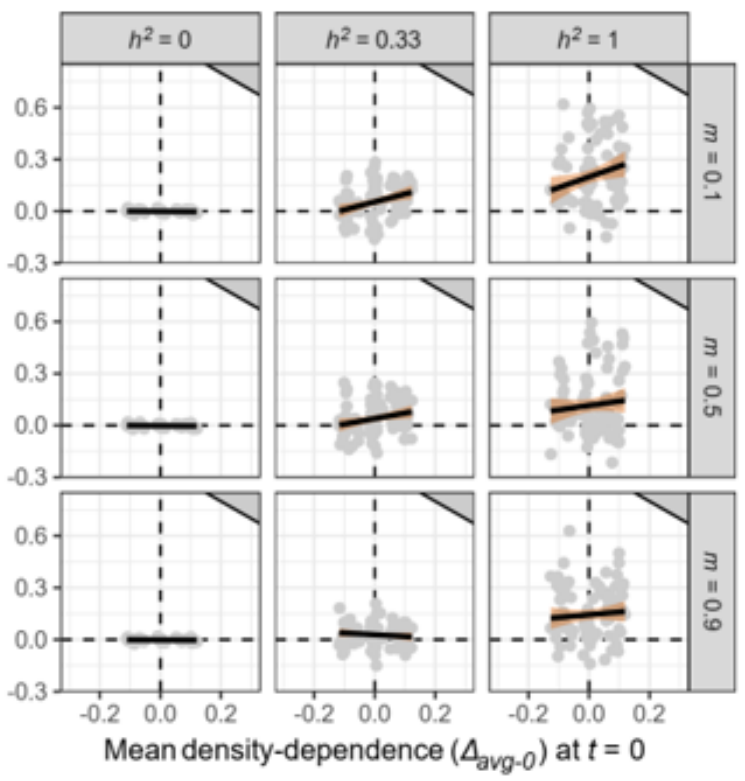


Figure 6. Evolutionary changes in density-dependence $\Delta_{a v g-0}$ at the range front as a function of

396 its initial value, sources of phenotypic variation and dispersal costs (case where $r_{0}=\log (5)$, see

397 Supplementary Figure S5.6 for the case where $\left.r_{0}=\log (1.5)\right)$. Grey dots are individual

398 replicates, with linear regression lines overlaid. Replicates with no individual variation in traits

399 are not displayed. Grey-filled areas indicate impossible phenotypes, more specifically evolved

400 values of $\Delta_{a v g-0}$ that would imply dispersal rates higher than the maximal possible $d_{\max }$ ( 1 if $401 d_{\max }$ is variable among individuals, 0.5 if it is not).

402

403 For both $d_{\max }$ and $\Delta_{a v g-0}$, inter-replicate variation in evolutionary outcomes tended to 404 increase the higher the initial trait heritability (Figs 5, 6).

\section{Sensitivity of results to growth rate}

406 Most of the qualitative results described above were recovered when growth rate was set to $407 \log (1.5)$ (Supplementary Material S5) instead of $\log (5)$. However, when fecundity is lower, we 408 find that:

409 - For genetic diversity, $R^{2}$ and AICc diverge in which model structure is best (density410 dependence included after evolution for the former, before evolution according to the $411 \quad$ latter; Supplementary Figures S5.1 and S5.2).

412 - When costs are high, high evolved values of $d_{0}$ can reduce expansion speeds 413 (Supplementary Figure S5.3).

$414 \quad$ - Dispersal capacity $d_{\max }$ now evolves to lower values when costs are high enough $(m=$ 415 0.9) instead of consistently evolving to higher values (Supplementary Figure S5.5).

416 - As a result, expansions become less density-dependent when costs are high and only $d_{\text {max }}$ can evolve (Supplementary Figure S5.6)

418 We also note that when $r_{0}=\log (1.5)$, there remains substantially more genetic variation in 419 traits after 100 generations than when $r_{0}=\log (5)$. However, the large majority of replicates 420 still has lost most of its genetic variation (Supplementary Material S4). 


\section{Discussion}

422 A large number of experimental and observational studies have described how dispersal is often

423 density-dependent, across a wide range of taxa (Harman et al., 2020; reviewed in Matthysen,

424 2005). In non-expanding metapopulations, many models have been developed to understand

425 under what conditions density-dependent vs density-independent dispersal evolves and is 426 maintained (e.g. Baines et al., 2020; Rodrigues \& Johnstone, 2014; Travis et al., 1999 among

427 many others). By contrast, until recently (Birzu et al., 2019), the implications of density428 dependent dispersal for the dynamics of pushed vs. pulled expansions remained mostly 429 unstudied, with research in that area focusing on Allee effects (e.g. Gandhi et al., 2016, 2019; 430 Hallatschek \& Nelson, 2008; Roques et al., 2012). In addition, although we know that dispersal 431 traits (including matching traits shaping density-dependence) can evolve during range 432 expansions (e.g. Chuang \& Peterson, 2016; Fronhofer et al., 2017; Mishra et al., 2020), we do 433 not know how sources of dispersal variation and dispersal evolution shape whether an 434 expansion stays or become pushed or pulled. We here used simulation models as a first attempt 435 to address this gap.

436 Information on traits post-evolution is needed to (more) accurately predict pushed expansion speed but not neutral diversity

438 We find that our simulations generally behave as expected from pushed expansion theory (see 439 e.g. Birzu et al., 2018, 2019), with some nuances however that may be key for its applicability in 440 more ecologically realistic contexts. Importantly, we found that expansion speed was best 441 predicted by the mean $d_{0}$ and the average density-dependence of dispersal after evolution (Fig. 442 2). The more dispersal increased with density, the more expansions advanced faster than 443 predicted from $d_{0}$ alone and were therefore pushed (Fig. 3), as expected from theory (Birzu et 444 al., 2019). Predictions based on $d_{0}$ only, or predictions ignoring evolution and using trait values 445 collected before the expansions performed consistently worse (Fig. 2). This is to some extent 446 unsurprising, as any equilibrium speed would only be reached after dispersal evolution has 447 occurred. However, this has practical implications: attempts to forecast expansions based on 448 traits collected in non-expanding regions of the range only are likely to mispredict velocities 
449 whenever dispersal evolves at the edge (which may be often, Chuang \& Peterson, 2016), unless 450 one is able to also predict dispersal evolutionary dynamics themselves (for this, see below).

451 Mortality costs strongly influenced the effect of dispersal traits on expansion speed (Fig. 3).

452 While it did not change the direction of effect, information about environmental context (in 453 particular connectivity) may be needed to make accurate predictions of the consequences of 454 density-dependence on expansion speed.

455 As predicted by theory (e.g. Birzu et al., 2019), neutral genetic diversity loss was slowed down 456 when dispersal was positively density-dependent (and therefore when expansions were pushed 457 based on velocity) (Fig. 4). However, this was only true when dispersal costs were low or 458 moderate. At high costs of dispersal, neutral diversity was actually lost slightly faster when 459 dispersal increased with density (Fig. 4). We recover here a key result we highlighted in previous 460 simulations, albeit without dispersal evolution (Dahirel, Bertin, Haond, et al., 2021): in some 461 contexts, the responses of genetic diversity and expansion velocity to density-dependence are 462 decoupled, and classifying an expansion as "pushed" based on one metric may not necessarily 463 mean it will be "pushed" based on the other. This contrasts in a way with previous theoretical 464 works implying that these two characteristics of pushed expansions are intrinsically linked (Birzu 465 et al., 2018). Increased costs of dispersal are known to lead to lower genetic diversity during 466 expansions (e.g. Mona et al., 2014). While we can expect that an increase in the number of 467 dispersers/size of founding propagules may compensate for this, the net effect may still be 468 negative if dispersal costs are too high (see e.g. Mona et al., 2014, for an example involving 469 long-distance dispersal rather than density-dependent dispersal). Again, pushed expansion 470 models that do not account for the diverse costs of dispersal (Bonte et al., 2012) in some way 471 may then overgeneralize. This would lead to predictions that do not apply as effectively in 472 contexts where costs of dispersal become important, for instance when species expand in highly 473 fragmented environments.

474 Interestingly and contrary to expansion velocity, the best models explaining neutral genetic 475 diversity loss used trait data collected at the start of the expansion, i.e. before trait evolution 476 (Fig. 2). We believe this mostly reflects specific implementation details of our individual-based 477 model, leading to generally fast loss of neutral diversity so starting dispersal traits had a 
478 disproportionate influence. Indeed, when fecundity was set to be lower, this discrepancy 479 between velocity and diversity vanished, with both better explained by traits post-evolution

480 (Supplementary Figures S5.1, S5.2). Further work is needed to see how reproduction and the 481 structure of genetic diversity (ploidy, number of loci considered, number of alleles per locus), by 482 influencing the baseline speed of diversity decay, can influence when dispersal traits have the 483 strongest effect on diversity.

484 Finally, we note that while accounting for the shape of the density-dispersal function often leads 485 to better predictions of the effect of density-dependence, models where the density486 dependence was estimated based only on dispersal at $N=0$ and $N=K$ still performed almost 487 as well and sometimes better (Fig. 2, Supplementary Figures S5.1, S5.2). If confirmed, this is 488 very interesting for future empirical studies. Indeed, it implies than most of the predictive 489 benefits accrued by accounting for density-dependence can be obtained from a relatively small 490 amount of dispersal data, even for pushed expansions.

491 Sources of variation and costs of dispersal determine when dispersal evolution leads pushed 492 expansions to become more pulled

493 Our simulations show that the strength and direction of the density-dependence seen at the 494 range edge after evolution is to some extent predictable, if we have information about 495 environmental characteristics (dispersal costs), initial trait values and sources of within496 individual variation (Fig. 6). There still remains substantial amounts of unexplained variation 497 (Fig. 6), especially at higher levels of initial heritability. This is in line with previous work showing 498 that evolutionary stochasticity and serial founder events can lead to high variability in 499 evolutionary outcomes and spread dynamics between initially identical or near-identical 500 expansions (Dallas et al., 2020; Phillips, 2015; Weiss-Lehman et al., 2017). As already discussed 501 by these authors and others, this puts an intrinsic limit on our ability to predict the evolutionary 502 trajectories and spread of individual range expansions before they start.

503 Generally speaking, we find that Erm and Phillips (2020)'s key prediction, that pushed 504 expansions tend to become pulled with time, is only fulfilled under a relatively narrow set of 505 conditions when pushed dynamics are caused by density-dependent dispersal, rather than Allee 
506 effects as in their study. Simulated expansions starting from pushed dynamics (positive 507 dispersal-density mean slope) only moved consistently towards more pulled dynamics when 508 heritability was high, mortality was low and there was no individual variation in dispersal 509 capacity i.e. $d_{\max }$ (Fig. 6). When this conditions were not fulfilled, pushed expansions tended to 510 stay pushed, and pulled expansions either stayed pulled or actually became pushed with time 511 (Fig. 6). This is slightly surprising as, as detailed in the Introduction, Travis et al (2009)'s 512 simulation study using the same dispersal function as ours concluded that dispersal become 513 overall unconditionally high when it evolves at range edges. Our results show that the direct 514 translation of that prediction into a prediction about pushed expansions, while intuitively 515 appealing, must be done with extreme care, if at all. We do confirm that dispersal become 516 overall less conditional at the edge (Supplementary Material S6). However, this must not be 517 seen as synonymous with "expansions become less pushed", as dispersal functions may be 518 flat/unconditional over a large part of the range of densities yet still show positive density519 dependence when compared to $d_{0}$ (see e.g. Fig. 1D).

520 We believe the divergence between Erm and Phillips (2020) and our simulations hinges on the 521 fact that the dispersal function is shaped by two types of parameters which can have conflicting 522 effects on the absolute density-dependence reached after evolution:

523 - When only dispersal capacity i.e. $d_{\max }$ can evolve, we find that pulled expansions 524 become more pulled, and pushed expansions more pushed with time when $d_{\max }$ 525 increases due to spatial sorting (Fig. 6B), and the reverse in the few cases where $d_{\max }$ decreases at the range edge (when high costs cannot be compensated by low fecundity, Supplementary Figures S5.5 and S5.6). As evolution leads to change in dispersal capacity along the range edge (Fig. 5), the absolute response to density can change even if the relative response does not (Fig. 6). Our results here contradict hypotheses put forward by Birzu et al. (2019), that evolutionary changes in dispersal motility would not influence "pushiness" since they would affect velocities the same way across the range of densities. We show here that this is not necessarily true. Increasing dispersal capacity increases the range of possible dispersal rates (Kun \& Scheuring, 2006) (Fig. 1), and as a result increases the maximal potential amplitude of the density-dependence. 
- When only dispersal matching traits $(\alpha$ and $\beta$ ) can evolve but not dispersal capacity, we find that initially pushed expansions are more likely to stay pushed when dispersal is more costly (Fig. 6A). This is fully in line with some aspects of existing dispersal theory, which predicts that higher costs of dispersal favour positive density-dependent dispersal strategies (Rodrigues \& Johnstone, 2014; Travis et al., 1999). This also reproduces qualitatively results from a previous study involving two of the authors (Dahirel, Bertin, Haond, et al., 2021; Dahirel, Bertin, Calcagno, et al., 2021). The microwasp low). Also, contrary to our initial hypotheses, we found that under some other contexts, evolution can lead to more, not less, positive density dependence in dispersal at range edges (Fig. 6A). That idea is also supported experimentally by at least one study (Mishra et al., 2020).

551 shape the overall response (Fig. 6). The net effect will, in reality, likely depend on each trait's

552 heritability, trait variability and environmental context. However, it may be that, contrary to

553 Erm and Phillips (2020) predictions for Allee effect-induced pushed expansions, pushed

554 expansions caused by density-dependent dispersal are "resistant" to evolutionary change at the

555 range edge in many circumstances. To be able to forecast if a given expansion will likely stay 556 pushed, with the associated implications in terms of velocity or genetic diversity, we will 557 therefore need better information about the degree of genetic variation in not only dispersal 558 capacity, but also dispersal reaction norms (Saastamoinen et al., 2018), information that is 559 currently lacking for many species, including expanding ones.

\section{Conclusion: towards even more realistic representations of phenotypic variation?}

561 Our simulation study shows that whether an expansion stays or become pushed during spatial 562 evolution is to some extent predictable from initial conditions, but information about dispersal 
563 costs (and probably more generally environmental conditions) is needed to make correct 564 predictions. Predictions also depend on whether individuals vary in dispersal capacity, relative 565 response to density, or both. While our model is arguably more complex than some of the 566 existing ones, especially due to the addition of evolution, it still makes some key simplifications, 567 which may need to be reexamined to determine whether they influence our results.

568 First, we used asexual haploid virtual organisms and each dispersal trait was shaped by one 569 locus only; evolutionary dynamics might be influenced by the reproductive system, or the 570 number of loci involved in inheritance and their genetic architecture (Saastamoinen et al., 571 2018). Recent simulations suggest that while these may influence the strength of evolutionary 572 change during expansion (and its effect on expansion velocity), they might not change the 573 overall direction of the evolutionary response (Weiss-Lehman \& Shaw, 2021). These results 574 remain however to be confirmed in the context of pushed expansions and density-dependent 575 dispersal. We also ignored mutation for simplicity and focused only on the fate of initial 576 standing variation. Work by Erm and Phillips (2020) suggests that adding mutation would merely 577 accelerate/accentuate trait differentiation compared to what we observed, as it would provide 578 variation allowing spatial sorting to continue.

579 Second and most critically, we only studied the effect of dispersal evolution. Whether or not an 580 expansion is pushed, and to what degree, depends on both dispersal and population growth 581 (Birzu et al., 2019), and the latter can be further subdivided in its fundamental life history 582 components, fecundity and survival. However, dispersal, fecundity and survival can all vary 583 among individuals, and can all be density-dependent at the same time, potentially in ways that 584 could cancel each other out. They may also be correlated in syndromes at the within- and/or 585 among-species level (Beckman et al., 2018; Guerra, 2011; Jacob et al., 2019; Ochocki et al., 586 2020; Ronce \& Clobert, 2012), and these syndromes may shape and constrain the evolution of 587 their constituent traits (Ronce \& Clobert, 2012; Wright et al., 2019), including during range 588 expansions (Ochocki et al., 2020; Urquhart \& Williams, 2021). In addition, theoretical work even 589 suggests that density fluctuations themselves are one of the root evolutionary drivers shaping 590 the (co)variation of life history traits, and potentially their association with behaviours such as 591 dispersal (Wright et al., 2019, 2020). How the complexities of phenotypic structure and trait 
592 coevolution drive the net effect of population density on spread, influencing the dynamics of

593 pushed vs pulled expansions and our ability to predict them, remain to be discovered.

\section{Data availability}

595 Netlogo model code and R scripts to reproduce all analyses presented in this manuscript are 596 available on Github (https://github.com/mdahirel/pushed-pulled-2020-heritability-IBM) and 597 archived in Zenodo (https://doi.org/10.5281/zenodo.5830993). Simulation outputs are also 598 archived in Zenodo (https://doi.org/10.5281/zenodo.5830996).

\section{Funding}

600 This work was funded by the French Agence Nationale de la Recherche (PushToiDeLa, 601 ANR-18-CE32-0008). CG holds a doctoral grant from the French Ministry of Higher Education 602 and Research.

\section{Author contributions}

604 Funding: EV; initial manuscript idea: MD and EV; simulation model development: MD and CG; 605 simulated data analysis: MD; initial manuscript draft: MD. All authors read and edited the 606 manuscript, and approved the final version.

\section{References}

608 Baines, C. B., Ferzoco, I. M. C., \& McCauley, S. J. (2019). Phenotype-by-environment interactions 609 influence dispersal. Journal of Animal Ecology, 88(8), 1263-1274. https://doi.org/10.1111/1365$610 \quad 2656.13008$

611 Baines, C. B., \& McCauley, S. J. (2018). Natal habitat conditions have carryover effects on 612 dispersal capacity and behavior. Ecosphere, 9(10), e02465. https://doi.org/10.1002/ecs2.2465

613 Baines, C. B., Travis, J. M. J., McCauley, S. J., \& Bocedi, G. (2020). Negative density-dependent 614 dispersal emerges from the joint evolution of density- and body condition-dependent dispersal 615 strategies. Evolution, 74(10), 2238-2249. https://doi.org/10.1111/evo.14085 
617 Beckman, N. G., Bullock, J. M., \& Salguero-Gómez, R. (2018). High dispersal ability is related to 618 fast life-history strategies. Journal of Ecology, 106(4), 1349-1362. https://doi.org/10.1111/1365$619 \quad 2745.12989$

620 Birzu, G., Hallatschek, O., \& Korolev, K. S. (2018). Fluctuations uncover a distinct class of 621 traveling waves. Proceedings of the National Academy of Sciences, 115(16), E3645-E3654. 622 https://doi.org/10.1073/pnas.1715737115

623 Birzu, G., Matin, S., Hallatschek, O., \& Korolev, K. S. (2019). Genetic drift in range expansions is 624 very sensitive to density dependence in dispersal and growth. Ecology Letters, 22(11), 1817625 1827. https://doi.org/10.1111/ele.13364

626 Bonte, D., Van Dyck, H., Bullock, J. M., Coulon, A., Delgado, M., Gibbs, M., Lehouck, V., 627 Matthysen, E., Mustin, K., Saastamoinen, M., Schtickzelle, N., Stevens, V. M., Vandewoestijne, 628 S., Baguette, M., Barton, K., Benton, T. G., Chaput-Bardy, A., Clobert, J., Dytham, C., ... Travis, J. 629 M. J. (2012). Costs of dispersal. Biological Reviews, 87(2), $290-312$. 630 https://doi.org/10.1111/j.1469-185X.2011.00201.x

631 Börger, L., \& Fryxell, J. (2012). Quantifying individual differences in dispersal using net squared 632 displacement. In J. Clobert, M. Baguette, T. G. Benton, \& J. M. Bullock (Eds.), Dispersal ecology 633 and evolution (pp. 222-230). Oxford University Press.

634 Bowler, D. E., \& Benton, T. G. (2005). Causes and consequences of animal dispersal strategies: 635 relating individual behaviour to spatial dynamics. Biological Reviews, 80(2), $205-225$. 636 https://doi.org/10.1017/S1464793104006645

637 Bürkner, P.-C., \& Vuorre, M. (2019). Ordinal regression models in psychology: a tutorial. 638 Advances in Methods and Practices in Psychological Science, (March 2019), 77-101. 639 https://doi.org/10.1177/2515245918823199

640 Christensen, R. H. B. (2019). ordinal-Regression models for ordinal data. https://CRAN.R641 project.org/package=ordinal 
642 Chuang, A., \& Peterson, C. R. (2016). Expanding population edges: theories, traits, and trade643 offs. Global Change Biology, 22(2), 494-512. https://doi.org/10.1111/gcb.13107

644 Cote, J., Bestion, E., Jacob, S., Travis, J., Legrand, D., \& Baguette, M. (2017). Evolution of 645 dispersal strategies and dispersal syndromes in fragmented landscapes. Ecography, 40(1), 56646 73. https://doi.org/10.1111/ecog.02538

647 Courchamp, F., Berec, L., \& Gascoigne, J. (2008). Allee effects in ecology and conservation. 648 Oxford University Press.

649 Dahirel, M., Bertin, A., Calcagno, V., Duraj, C., Fellous, S., Groussier, G., Lombaert, E., Mailleret, 650 L., Marchand, A., \& Vercken, E. (2021). Landscape connectivity alters the evolution of density651 dependent dispersal during pushed range expansions. bioRxiv (Peer-Reviewed and 652 Recommended by Peer Community In Evolutionary Biology), 2021.03.03.433752. 653 https://doi.org/10.1101/2021.03.03.433752

654 Dahirel, M., Bertin, A., Haond, M., Blin, A., Lombaert, E., Calcagno, V., Fellous, S., Mailleret, L., 655 Malausa, T., \& Vercken, E. (2021). Shifts from pulled to pushed range expansions caused by 656 reduction of landscape connectivity. Oikos, 130(5), 708-724. https://doi.org/10.1111/oik.08278

657 Dallas, T., Melbourne, B., \& Hastings, A. (2020). Community context and dispersal stochasticity 658 drive variation in spatial spread. Journal of Animal Ecology, 89(11), 2657-2664. 659 https://doi.org/10.1111/1365-2656.13331

660 Erm, P., \& Phillips, B. L. (2020). Evolution transforms pushed waves into pulled waves. The 661 American Naturalist, 195(3), E87-E99. https://doi.org/10.1086/707324

662 Fronhofer, E. A., Gut, S., \& Altermatt, F. (2017). Evolution of density-dependent movement 663 during experimental range expansions. Journal of Evolutionary Biology, 30(12), 2165-2176. 664 https://doi.org/10.1111/jeb.13182

665 Gandhi, S. R., Korolev, K. S., \& Gore, J. (2019). Cooperation mitigates diversity loss in a spatially 666 expanding microbial population. Proceedings of the National Academy of Sciences, 116(47), 667 23582-23587. https://doi.org/10.1073/pnas.1910075116 
668 Gandhi, S. R., Yurtsev, E. A., Korolev, K. S., \& Gore, J. (2016). Range expansions transition from 669 pulled to pushed waves as growth becomes more cooperative in an experimental microbial 670 population. Proceedings of the National Academy of Sciences, 113(25), 6922-6927. 671 https://doi.org/10.1073/pnas.1521056113

672 Gregory, S. D., Bradshaw, C. J. A., Brook, B. W., \& Courchamp, F. (2010). Limited evidence for the 673 demographic Allee effect from numerous species across taxa. Ecology, 91(7), 2151-2161. 674 https://doi.org/10.1890/09-1128.1

675 Grimm, V., Berger, U., DeAngelis, D. L., Polhill, J. G., Giske, J., \& Railsback, S. F. (2010). The ODD 676 protocol: a review and first update. Ecological Modelling, 221(23), 2760-2768. 677 https://doi.org/10.1016/j.ecolmodel.2010.08.019

678 Grimm, V., Railsback, S. F., Vincenot, C. E., Berger, U., Gallagher, C., DeAngelis, D. L., Edmonds, 679 B., Ge, J., Giske, J., Groeneveld, J., Johnston, A. S. A., Milles, A., Nabe-Nielsen, J., Polhill, J. G., 680 Radchuk, V., Rohwäder, M.-S., Stillman, R. A., Thiele, J. C., \& Ayllón, D. (2020). The ODD protocol 681 for describing agent-based and other simulation models: a second update to improve clarity, 682 replication, and structural realism. Journal of Artificial Societies and Social Simulation, 23(2), 7.

683 Guerra, P. A. (2011). Evaluating the life-history trade-off between dispersal capability and 684 reproduction in wing dimorphic insects: a meta-analysis. Biological Reviews, 86(4), 813-835. 685 https://doi.org/10.1111/j.1469-185X.2010.00172.x

686 Hallatschek, O., \& Korolev, K. S. (2009). Fisher waves in the strong noise limit. Physical Review 687 Letters, 103(10), 108103. https://doi.org/10.1103/PhysRevLett.103.108103

688 Hallatschek, O., \& Nelson, D. R. (2008). Gene surfing in expanding populations. Theoretical 689 Population Biology, 73(1), 158-170. https://doi.org/10.1016/j.tpb.2007.08.008

690 Haond, M., Morel-Journel, T., Lombaert, E., Vercken, E., Mailleret, L., \& Roques, L. (2018). When 691 higher carrying capacities lead to faster propagation. bioRxiv (Peer-Reviewed and Recommended 692 by Peer Community In Ecology), 307322. https://doi.org/10.1101/307322 
693 Harman, R. R., Goddard, J., Shivaji, R., \& Cronin, J. T. (2020). Frequency of occurrence and 694 population-dynamic consequences of different forms of density-dependent emigration. The 695 American Naturalist, 195(5), 851-867. https://doi.org/10.1086/708156

696 Hassell, M. P., Lawton, J. H., \& May, R. M. (1976). Patterns of dynamical behaviour in single697 species populations. Journal of Animal Ecology, 45(2), 471-486. https://doi.org/10.2307/3886

698 Jacob, S., Chaine, A. S., Huet, M., Clobert, J., \& Legrand, D. (2019). Variability in dispersal 699 syndromes is a key driver of metapopulation dynamics in experimental microcosms. The 700 American Naturalist, 194(5), 613-626. https://doi.org/10.1086/705410

701 Kun, Á., \& Scheuring, I. (2006). The evolution of density-dependent dispersal in a noisy spatial 702 population model. Oikos, 115(2), 308-320. https://doi.org/10.1111/j.2006.0030-1299.15061.x

703 Lewis, M., Petrovskii, S. V., \& Potts, J. (2016). The mathematics behind biological invasions. 704 Springer International Publishing. https://doi.org/10.1007/978-3-319-32043-4

705 Little, C. J., Fronhofer, E. A., \& Altermatt, F. (2019). Dispersal syndromes can impact ecosystem 706 functioning in spatially structured freshwater populations. Biology Letters, 15(3), 20180865. 707 https://doi.org/10.1098/rsbl.2018.0865

708 Matthysen, E. (2012). Multicausality of dispersal: a review. In J. Clobert, M. Baguette, T. G. 709 Benton, \& J. M. Bullock (Eds.), Dispersal ecology and evolution (pp. 3-18). Oxford University 710 Press.

711 Matthysen, E. (2005). Density-dependent dispersal in birds and mammals. Ecography, 28(3), 712 403-416. https://doi.org/10.1111/j.0906-7590.2005.04073.x

713 Miller, T. E. X., Angert, A. L., Brown, C. D., Lee-Yaw, J. A., Lewis, M., Lutscher, F., Marculis, N. G., 714 Melbourne, B. A., Shaw, A. K., Szűcs, M., Tabares, O., Usui, T., Weiss-Lehman, C., \& Williams, J. L. 715 (2020). Eco-evolutionary dynamics of range expansion. Ecology, 101(10), e03139. 716 https://doi.org/10.1002/ecy.3139 
717 Mishra, A., Chakraborty, P. P., \& Dey, S. (2020). Dispersal evolution diminishes the negative

718 density dependence in dispersal. Evolution, evo.14070. https://doi.org/10.1111/evo.14070

719 Mona, S., Ray, N., Arenas, M., \& Excoffier, L. (2014). Genetic consequences of habitat 720 fragmentation during a range expansion. Heredity, 112(3), 291-299.

721 https://doi.org/10.1038/hdy.2013.105

722 Ochocki, B. M., \& Miller, T. E. X. (2017). Rapid evolution of dispersal ability makes biological 723 invasions faster and more variable. Nature Communications, 8, 14315. 724 https://doi.org/10.1038/ncomms14315

725 Ochocki, B. M., Saltz, J. B., \& Miller, T. E. X. (2020). Demography-dispersal trait correlations 726 modify the eco-evolutionary dynamics of range expansion. The American Naturalist, 195(2), 727 231-246. https://doi.org/10.1086/706904

728 Pedersen, T. L. (2020). patchwork: the composer of plots. https://CRAN.R729 project.org/package=patchwork

730 Phillips, B. L. (2015). Evolutionary processes make invasion speed difficult to predict. Biological 731 Invasions, 17(7), 1949-1960. https://doi.org/10.1007/s10530-015-0849-8

732 Phillips, B. L., Brown, G. P., Webb, J. K., \& Shine, R. (2006). Invasion and the evolution of speed 733 in toads. Nature, 439(7078), 803-803. https://doi.org/10.1038/439803a

734 Phillips, B. L., \& Perkins, T. A. (2019). Spatial sorting as the spatial analogue of natural selection. 735 Theoretical Ecology. https://doi.org/10.1007/s12080-019-0412-9

736 R Core Team. (2021). R: a language and environment for statistical computing. R Foundation for 737 Statistical Computing. https://www.R-project.org/

738 Rodrigues, A. M. M., \& Johnstone, R. A. (2014). Evolution of positive and negative density739 dependent dispersal. Proceedings of the Royal Society of London B: Biological Sciences, 740 281(1791), 20141226. https://doi.org/10.1098/rspb.2014.1226 
741 Ronce, O., \& Clobert, J. (2012). Dispersal syndromes. In J. Clobert, M. Baguette, T. G. Benton, \&

742 J. M. Bullock (Eds.), Dispersal ecology and evolution (pp. 119-138). Oxford University Press.

743 Roques, L., Garnier, J., Hamel, F., \& Klein, E. K. (2012). Allee effect promotes diversity in

744 traveling waves of colonization. Proceedings of the National Academy of Sciences, 109(23),

745 8828-8833. https://doi.org/10.1073/pnas.1201695109

746 Saastamoinen, M., Bocedi, G., Cote, J., Legrand, D., Guillaume, F., Wheat, C. W., Fronhofer, E. A.,

747 Garcia, C., Henry, R., Husby, A., Baguette, M., Bonte, D., Coulon, A., Kokko, H., Matthysen, E., 748 Niitepõld, K., Nonaka, E., Stevens, V. M., Travis, J. M. J., ... Delgado, M. del M. (2018). Genetics of 749 dispersal. Biological Reviews, 93(1), 574-599. https://doi.org/10.1111/brv.12356

750 Salecker, J., Sciaini, M., Meyer, K. M., \& Wiegand, K. (2019). The nlrx R package: a next751 generation framework for reproducible NetLogo model analyses. Methods in Ecology and 752 Evolution, 10(11), 1854-1863. https://doi.org/10.1111/2041-210X.13286

753 Shine, R., Brown, G. P., \& Phillips, B. L. (2011). An evolutionary process that assembles 754 phenotypes through space rather than through time. Proceedings of the National Academy of 755 Sciences, 108(14), 5708-5711. https://doi.org/10.1073/pnas.1018989108

756 Simmons, Adam D., \& Thomas, Chris D. (2004). Changes in dispersal during species' range 757 expansions. The American Naturalist, 164(3), 378-395. https://doi.org/10.1086/423430

758 Simpson, G. L. (2021). gratia: Graceful "ggplot"-Based graphics and other functions for GAMs 759 fitted using "mgcv". https://CRAN.R-project.org/package=gratia

760 Stokes, A. N. (1976). On two types of moving front in quasilinear diffusion. Mathematical 761 Biosciences, 31(3), 307-315. https://doi.org/10.1016/0025-5564(76)90087-0

762 Szúcs, M., Melbourne, B. A., Tuff, T., Weiss-Lehman, C., \& Hufbauer, R. A. (2017). Genetic and 763 demographic founder effects have long-term fitness consequences for colonising populations. 764 Ecology Letters, 20(4), 436-444. https://doi.org/10.1111/ele.12743 
765 Travis, J. M. J., Murrell, D. J., \& Dytham, C. (1999). The evolution of density-dependent

766 dispersal. Proceedings of the Royal Society of London B: Biological Sciences, 266(1431), 1837-

767 1842. https://doi.org/10.1098/rspb.1999.0854

768 Travis, J. M. J., Mustin, K., Benton, T. G., \& Dytham, C. (2009). Accelerating invasion rates result

769 from the evolution of density-dependent dispersal. Journal of Theoretical Biology, 259(1), 151-

770 158. https://doi.org/10.1016/j.jtbi.2009.03.008

771 Tutz, G., \& Schmid, M. (2016). Modeling discrete time-to-event data. Springer International 772 Publishing. https://doi.org/10.1007/978-3-319-28158-2

773 Urquhart, C. A., \& Williams, J. L. (2021). Trait correlations and landscape fragmentation jointly 774 alter expansion speed via evolution at the leading edge in simulated range expansions. 775 Theoretical Ecology. https://doi.org/10.1007/s12080-021-00503-z

776 Van Petegem, K., Moerman, F., Dahirel, M., Fronhofer, E. A., Vandegehuchte, M. L., Van 777 Leeuwen, T., Wybouw, N., Stoks, R., \& Bonte, D. (2018). Kin competition accelerates 778 experimental range expansion in an arthropod herbivore. Ecology Letters, 21(2), 225-234. 779 https://doi.org/10.1111/ele.12887

780 Wang, C.-H., Matin, S., George, A. B., \& Korolev, K. S. (2019). Pinned, locked, pushed, and pulled 781 traveling waves in structured environments. Theoretical Population Biology, 127, 102-119. 782 https://doi.org/10.1016/j.tpb.2019.04.003

783 Weiss-Lehman, C., Hufbauer, R. A., \& Melbourne, B. A. (2017). Rapid trait evolution drives 784 increased speed and variance in experimental range expansions. Nature Communications, 8, 785 14303. https://doi.org/10.1038/ncomms14303

786 Weiss-Lehman, C., \& Shaw, A. (2021). Contrasting effects of ecological and evolutionary 787 processes on range expansions and shifts. Authorea Preprint. 788 https://doi.org/10.22541/au.161217810.03338053/v1

789 Wickham, H., Averick, M., Bryan, J., Chang, W., McGowan, L., François, R., Grolemund, G., 790 Hayes, A., Henry, L., Hester, J., Kuhn, M., Pedersen, T., Miller, E., Bache, S., Müller, K., Ooms, J., 
791 Robinson, D., Seidel, D., Spinu, V., ... Yutani, H. (2019). Welcome to the Tidyverse. Journal of

792 Open Source Software, 4(43), 1686. https://doi.org/10.21105/joss.01686

793 Wilensky, U. (1999). NetLogo. Center for Connected Learning and Computer-Based Modeling,

794 Northwestern University. http://ccl.northwestern.edu/netlogo/

795 Williams, J. L., \& Levine, Jonathan M. (2018). Experimental evidence that density dependence

796 strongly influences plant invasions through fragmented landscapes. Ecology, 99(4), 876-884.

797 https://doi.org/10.1002/ecy.2156

798 Wood, S. N. (2017). Generalized additive models: An introduction with R (2nd ed.). Chapman and 799 Hall/CRC.

800 Wright, J., Bolstad, G. H., Araya-Ajoy, Y. G., \& Dingemanse, N. J. (2019). Life-history evolution 801 under fluctuating density-dependent selection and the adaptive alignment of pace-of-life 802 syndromes. Biological Reviews, 94(1), 230-247. https://doi.org/10.1111/brv.12451

803 Wright, J., Solbu, E. B., \& Engen, S. (2020). Contrasting patterns of density-dependent selection 804 at different life stages can create more than one fast-slow axis of life-history variation. Ecology 805 and Evolution, 10(6), 3068-3078. https://doi.org/10.1002/ece3.6122 
expansion velocity

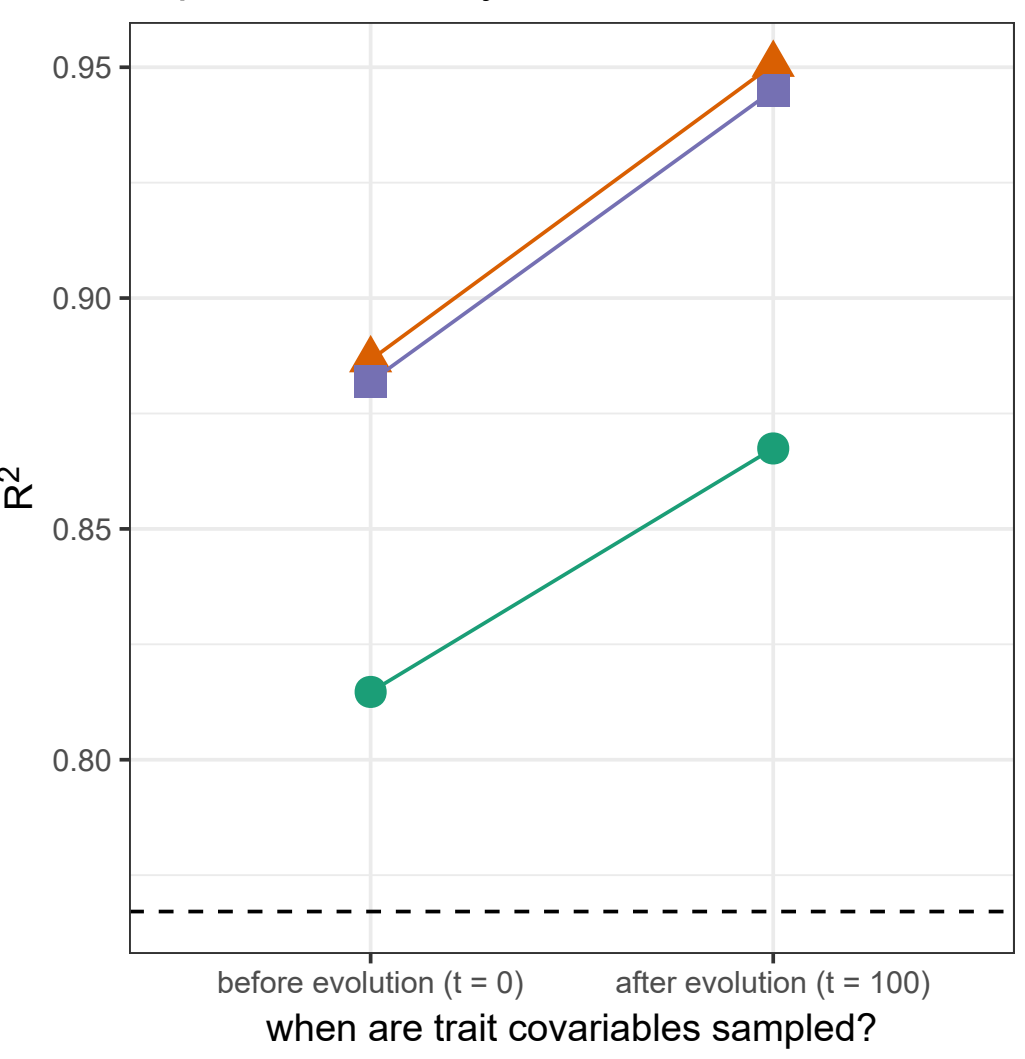

neutral diversity

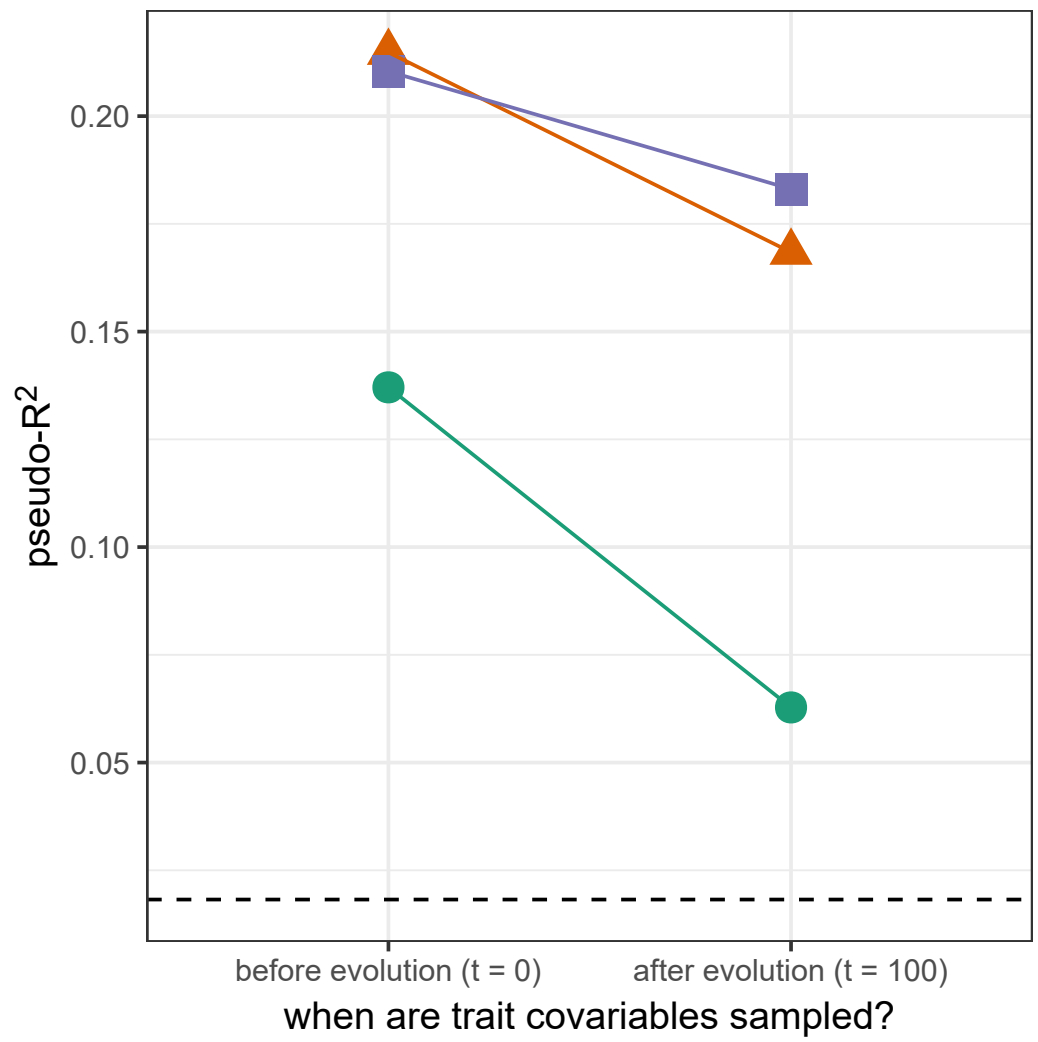

Density dependence? ignored

$\_\Delta_{\text {avg-0 }}$ (shape-dep.)

- $\Delta_{K-0}$ (shape-indep.) 

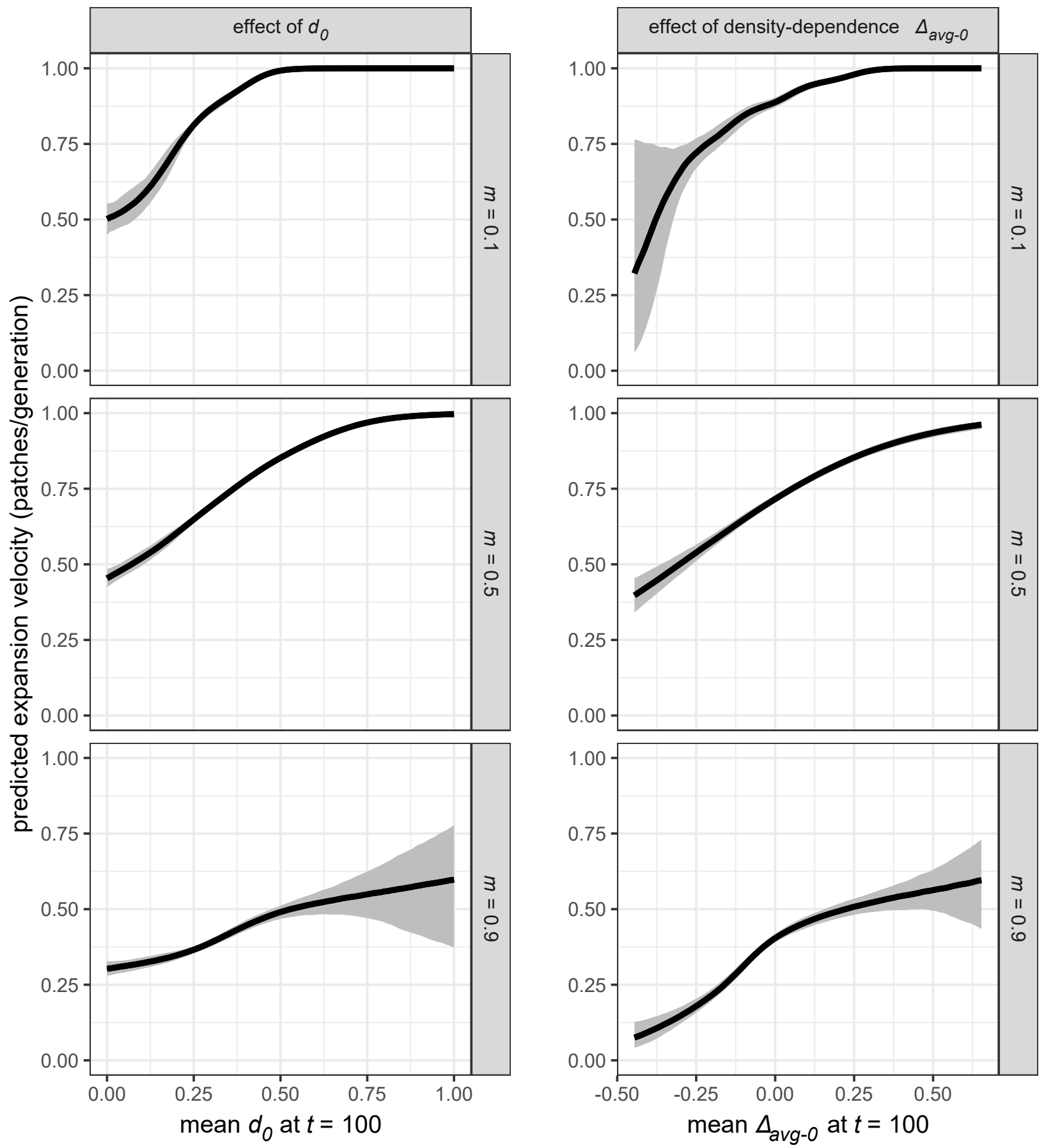

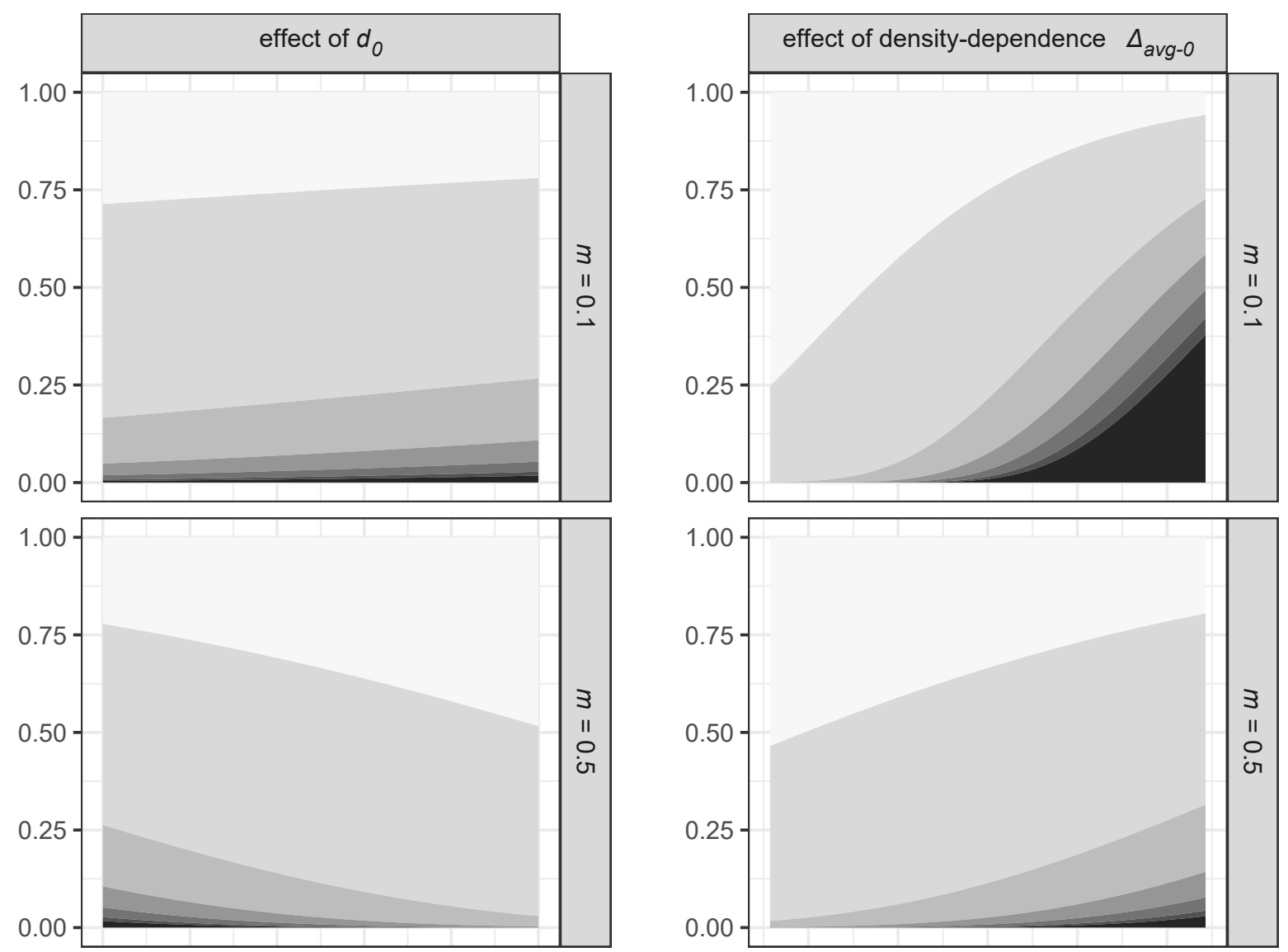

generations before allele fixation:
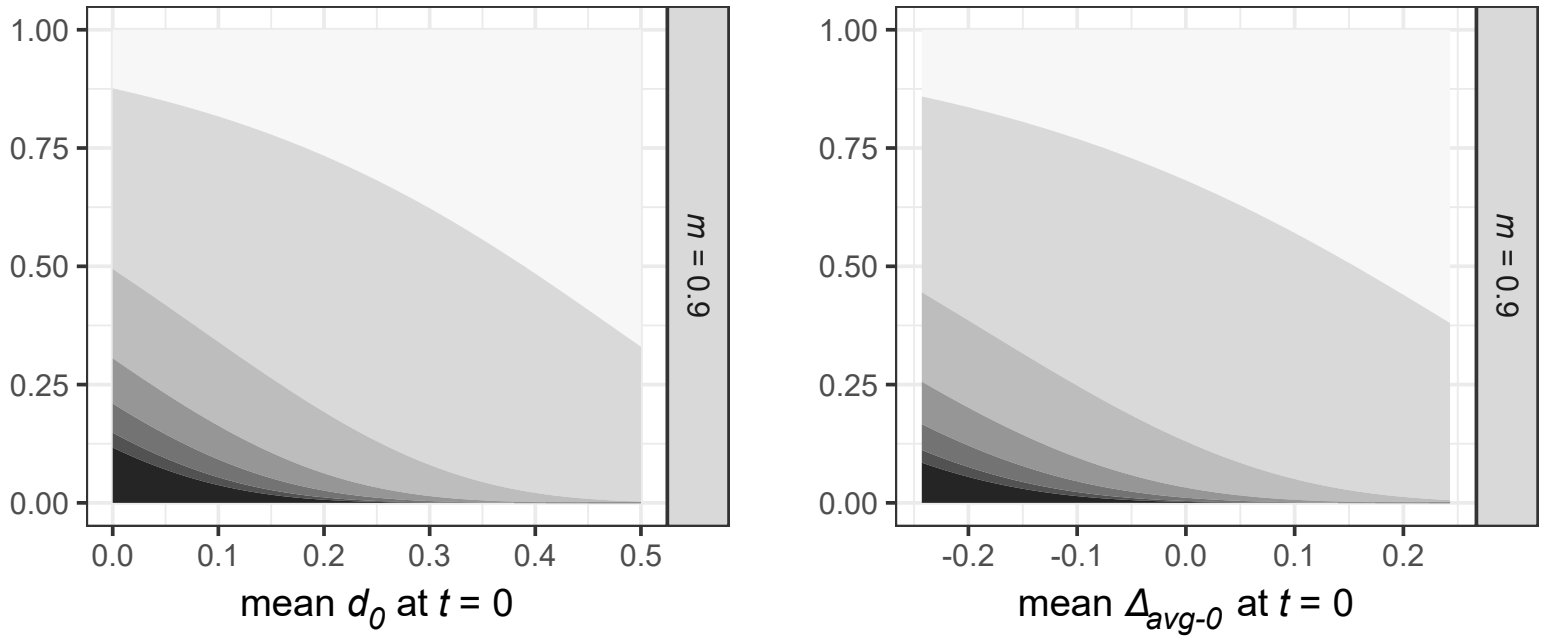

$\leq 20$

21 to 40

41 to 60

61 to 80

81 to 100

101 to 120

$>120$ 


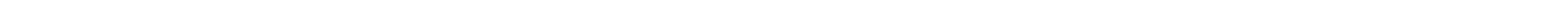


A: Individual variation in $\alpha$ and $\beta$
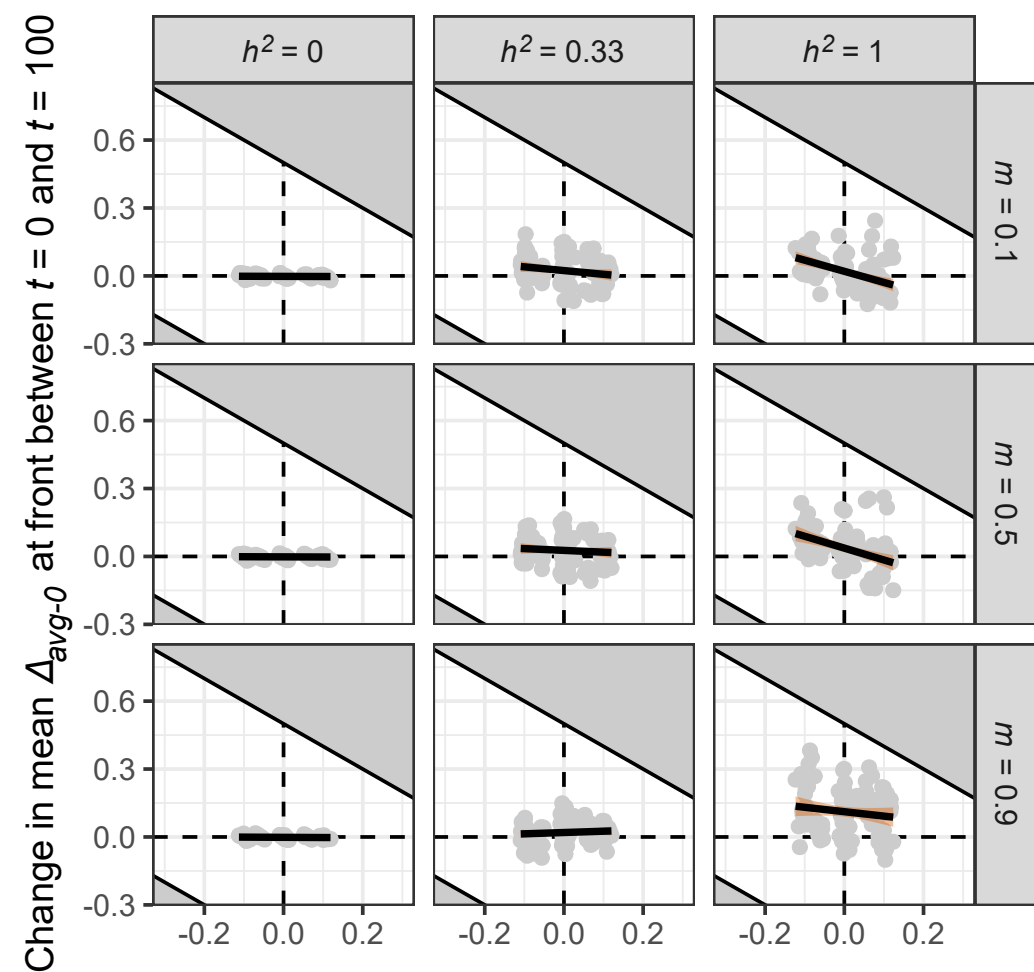

$\mathrm{B}$ : Individual variation in $d_{\max }$

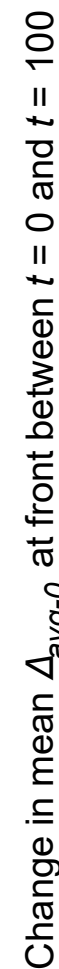
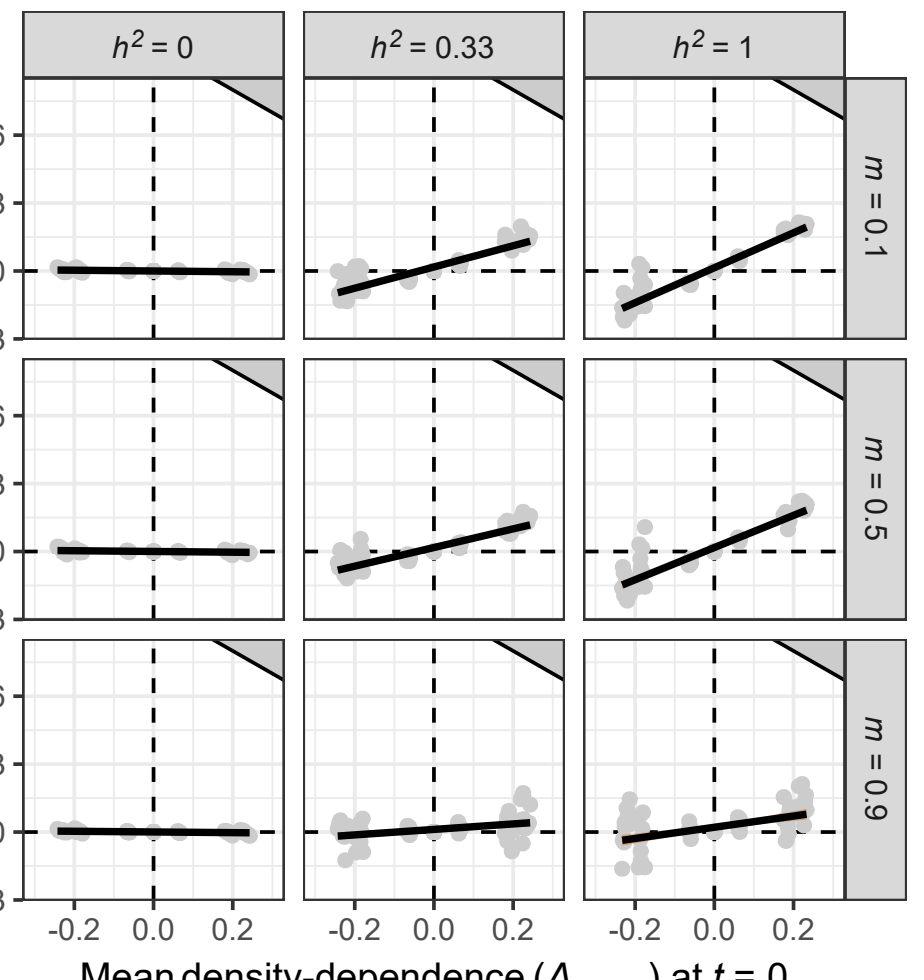

Mean density-dependence $\left(\Delta_{\text {avg-0 }}\right)$ at $t=0$

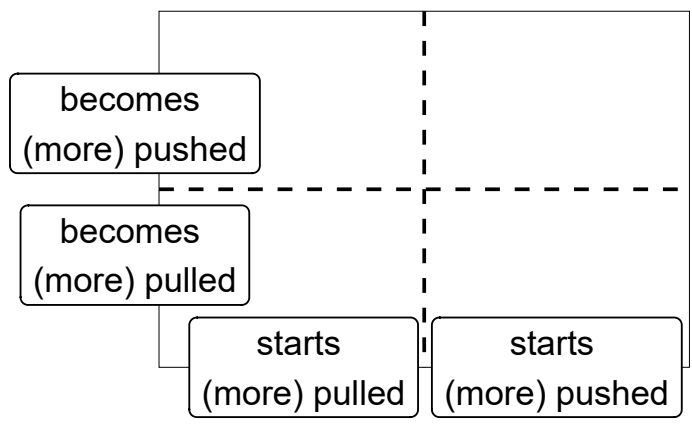

C: Individual variation in $\alpha, \beta$, and $d_{\max }$

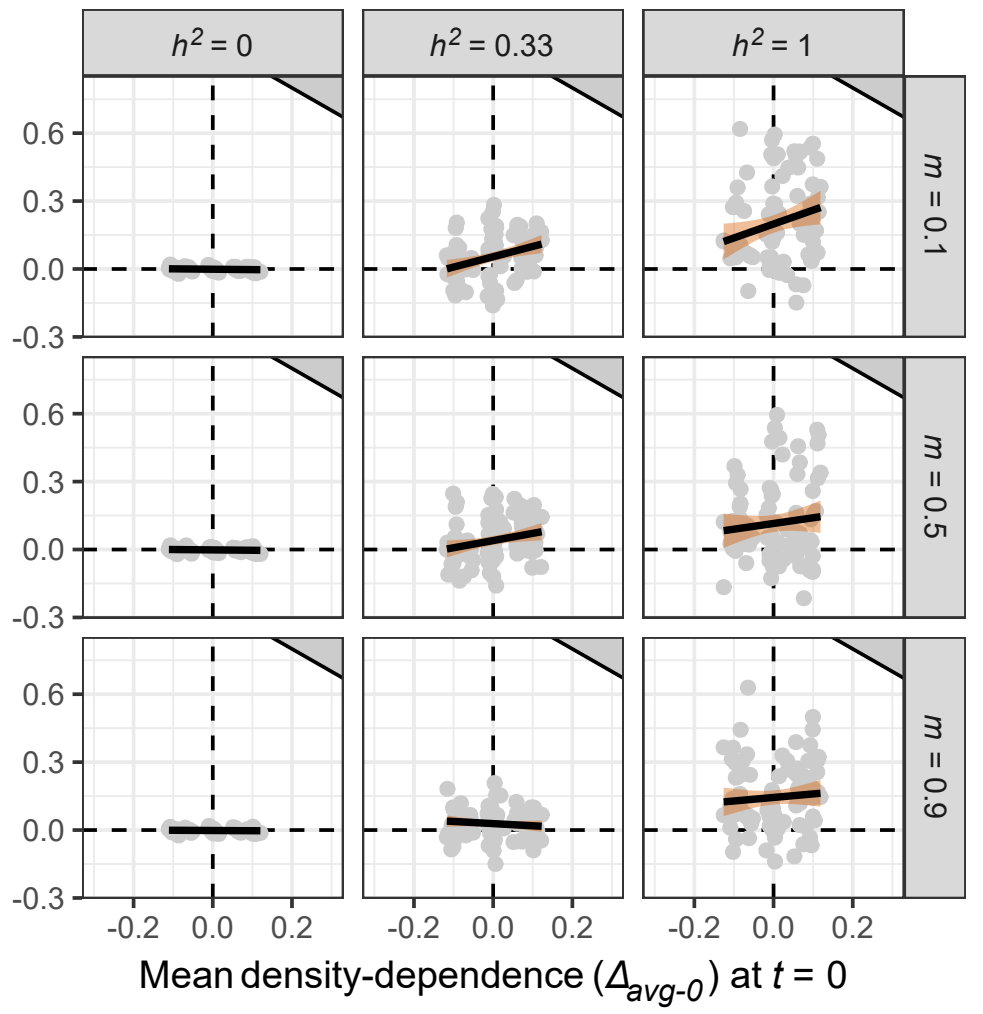

